\title{
Age-dependent expression changes of circadian system-related genes reveal a potentially conserved link to aging
}

\author{
Emanuel Barth ${ }^{1}$, Akash Srivastava ${ }^{1,2,3}$, Diane Wengerodt ${ }^{3}$, Milan Stojiljkovic ${ }^{3}$, Hubertus Axer ${ }^{3}$, \\ Otto W. Witte ${ }^{3}$, Alexandra Kretz ${ }^{3,{ }^{*}}$, Manja Marz ${ }^{1,2,4,5, *}$ \\ ${ }^{1}$ Bioinformatics/High Throughput Analysis, Faculty of Mathematics and Computer Science, Friedrich Schiller \\ University Jena, Jena, Germany \\ ${ }^{2} \mathrm{FLI}$ Leibniz Institute for Age Research, Jena, Germany \\ ${ }^{3}$ Hans Berger Department of Neurology, Jena University Hospital, Jena, Germany \\ ${ }^{4}$ German Center for Integrative Biodiversity Research (iDiv), Halle-Jena-Leipzig, Germany \\ ${ }^{5}$ European Virus Bioinformatics Center (EVBC), Jena, Germany \\ *Shared last authorships
}

Correspondence to: Emanuel Barth; email: emanuel.barth@uni-jena.de

Keywords: aging, circadian clock system, circadian rhythm, inter-species comparison, longevity, RNA-Seq

Received: March 16, $2021 \quad$ Accepted: December 1, $2021 \quad$ Published: December 19, 2021

Copyright: (C) 2021 Barth et al. This is an open access article distributed under the terms of the Creative Commons Attribution License (CC BY 3.0), which permits unrestricted use, distribution, and reproduction in any medium, provided the original author and source are credited.

\section{ABSTRACT}

The circadian clock system influences the biology of life by establishing circadian rhythms in organisms, tissues, and cells, thus regulating essential biological processes based on the day/night cycle. Circadian rhythms change over a lifetime due to maturation and aging, and disturbances in the control of the circadian system are associated with several age-related pathologies.

However, the impact of chronobiology and the circadian system on healthy organ and tissue aging remains largely unknown. Whether aging-related changes of the circadian system's regulation follow a conserved pattern across different species and tissues, hence representing a common driving force of aging, is unclear.

Based on a cross-sectional transcriptome analysis covering 329 RNA-Seq libraries, we provide indications that the circadian system is subjected to aging-related gene alterations shared between evolutionarily distinct species, such as Homo sapiens, Mus musculus, Danio rerio, and Nothobranchius furzeri. We discovered differentially expressed genes by comparing tissue-specific transcriptional profiles of mature, aged, and old-age individuals and report on six genes (per2, dec2, cirp, klf10, nfil3, and dbp) of the circadian system, which show conserved aging-related expression patterns in four organs of the species examined. Our results illustrate how the circadian system and aging might influence each other in various tissues over a long lifespan and conceptually complement previous studies tracking short-term diurnal and nocturnal gene expression oscillations.

\section{INTRODUCTION}

The life of most organisms is, beyond other influences, controlled by two central biological events: Biological rhythms based on the timely regulation of physiological processes, and the biology of aging. Circadian rhythms (CRs) are established by a genetically encoded circadian program, controlling a multitude of biological cycles and enabling an individual to adjust to periodic environmental changes during daytime, seasons, and lifetime. Thus, its conserved nature allows to synchronize metabolic, endocrine, behavioral, and complex intracellular events across a 24-hour day/night cycle (for a recent review, see [1]). 
Aging is an equally complex process, which is affected by a plethora of exogenous and endogenous factors, and which impacts virtually all crucial biological processes by a progressive loss of cellular functions $[2,3]$. It is well established that aging interferes with the regulation of the circadian system, which, in return, contributes to the manifestation and progression of aging-related diseases (reviewed in [4, 5]). Furthermore, ageindependent alterations of the circadian system can result in premature aging of vertebrates and invertebrates, suggesting a direct mutual interrelation between both processes [6].

As superordinate pacemaker of the circadian system in mammals, the suprachiasmatic nucleus ( $\mathrm{SCN}$ ) of the anterior hypothalamus imposes CRs on gene expression in each tissue and cell type. Thus, it conveys rhythmicity to all essential processes of physiology and behavior. In parallel with whole-body circadian rhythmicity imprinted by the SCN, circadian oscillations are regulated autonomously at the organ and cellular levels, with a functional clock residing in peripheral tissues and cell types. In comparison with mammals, including humans, CRs in lower vertebrates, such as fish, are less hierarchically organized and do not depend on the SCN [7]. Accordingly, the entrainment of CRs via environmental and endogenous cues is more potent in fish, where organs such as the skin and liver directly respond to light exposure and hormonal and nutritive conditions.

The functionality of the CRs extenuates with aging, generally manifesting in a lower penetrance of their rhythmicity. This decline is characterized by reduced amplitudes and increased scatter in circadian acrophases, disturbances of cell and tissue synchronization, and phase shifts in oscillations over a 24-hour cycle [8-10]. Likewise, fragmentation and shifts in the periodicity of CRs are prominent in elder mice and humans $[9,10]$, where they influence restactivity and sleep-wake period patterns. In addition to causing sleep disorders, disturbances in CRs are associated with pronounced stress responses, impaired DNA repair, and cancer. They are considered an independent risk factor for age-related disorders such as type II diabetes mellitus, Alzheimer's dementia, coronary heart disease, and tumors [11-14].

Furthermore, CR misalignment is associated with a loss in protein homeostasis, which, in turn, represents a common feature of various age-related proteinopathies, particularly those manifesting in the nervous system [15]. Accordingly, genes with a role in the surveillance and orchestration of CR signatures are essential in the control of biological processes at the individual cell and complex network levels, as they influence cellular maintenance, chromatin integrity and DNA repair, epigenetic modifications, autophagy, metabolic homeostasis, and immune functions. All these functions are fundamentally involved in the processes of cellular senescence and tissue aging [2]. Recent analyses in mammals reported that at least $40 \%$ of the protein-coding genes are subjected to circadian oscillations, and those genes correlated with agerelated dysfunctions are intimately connected to $\mathrm{CR}$ drivers [11].

However, it is not well studied in how far changes in the expression of CR core genes and their downstream targets are responsible for age-related variations in CR regulation. Moreover, it is unclear to what extent these changes in $\mathrm{CR}$ gene regulatory patterns may overlap in distinct tissues and species. A related open question remains as to whether a conserved age-related pattern of $\mathrm{CR}$ gene expression alterations might exist that will be common to and retraceable across different species and tissues. Apart from the pulsed expression of the superordinate core set of clock genes and related transcription factors in the brain hypothalamus [16], the aforementioned SCN-independent, self-sustained functionality of the CR is operating at the single-organ level, as it is well-described, e.g., for skin and liver [17]. Thus, evidence of SCN-independent CR regulation requires species with an evolutionary conserved decentralized CR, such as fish, and the inclusion of selfentrained organs.

In this study, we performed inter-species and interorgan transcriptional analyses based on 329 RNASeq libraries to identify CR regulatory patterns in the course of aging. We identified differentially expressed genes (DEGs) by assessing the transcriptional profiles of mature, aged, and old-age individuals. We compared these results between Homo sapiens (H. sapiens), Mus musculus (M. musculus), Danio rerio (D. rerio), and Nothobranchius furzeri ( $N$. furzeri), with the latter representing the family of extremely short-lived killifish, which is characterized by special adaption to ephemeral humidity of their habitats. The organs examined in this cross-sectional study comprised the brain, blood, liver, and skin.

We report 6 CR-related genes, i.e., per2, dec2, cirp, $k l f 10, n f i l 3$, and $d b p$, to exhibit conserved aging-related expression characteristics in up to 4 tissues of the species investigated. Additionally, we describe their role in tissue-inherent $\mathrm{CR}$ regulation and link them to age-associated processes and diseases. Our observations extend the understanding of the complex interplay between the circadian system and aging within vertebrates and provide a basis for further research on aging intervention strategies. 


\section{MATERIALS AND METHODS}

\section{Genomic and high-throughput transcriptomic data acquisition}

The genomes and annotations of $H$. sapiens, $M$. musculus and $D$. rerio used in this study were obtained from the Ensembl database (release version 92) [18]. For $N$. furzeri, the most recently published version of its genome assembly and the corresponding annotation were used $[19,20]$. The RNA-Seq data analyzed within this study were initially published by Irizar et al. [21] and compiled by the JenAge Consortium (http://www.jenage.de/). The whole RNA-Seq dataset is available at NCBI's Gene Expression Omnibus (H. sapiens: GSE75337, GSE103232; M. musculus: GSE75192, GSE78130; D. rerio: GSE74244 and N. furzeri: GSE52462, GSE66712). All human donors were classified into the following age groups: $24-29$ years, $60-65$ years, and 75-79 years $(n=$ 14-15 for each category). For mice, the selected age groups were 9 months, 24 months, and 30 months, with blood and skin samples deriving from identical individuals. Liver and brain sampling was based on a separate subset of animals (blood, skin, liver, $n=5$ for each; brain, $n=8$ ). Tissues extracted from fish, i.e., $D$. rerio and $N$. furzeri comprised brain, liver, and skin $(n=$ 5 for each) and were isolated at 12 months, 36 months, and 42 months and 12 weeks, 27 weeks, and 39 weeks of age, respectively (Figure 1). A total of 329 RNA-Seq libraries was examined. Within each species, the samples analyzed were collected at the same time of day. Further details regarding tissue sampling and extraction are available in the online supplement (https://osf.io/tkanf/).

\section{Lifespan normalization and transcriptomic data processing}

The 4 investigated species differ with respect to their lifespans. To facilitate comparability, we normalized

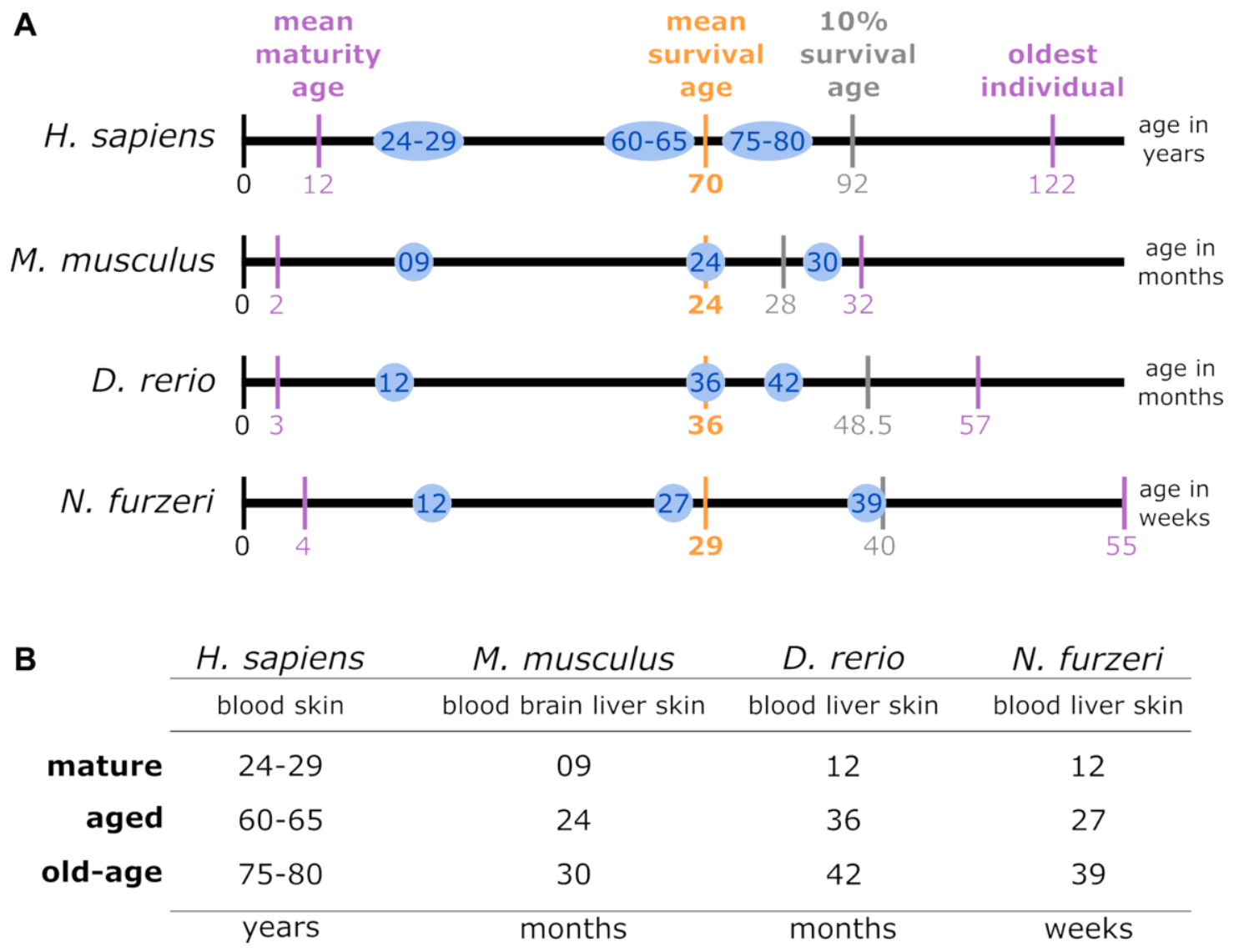

Figure 1. (A) Lifespan comparison: to align the species-specific chronological ages to biological age categories comparable between different species, the total individual's lifetime, represented by the length of the lifetime axis, was subdivided by index stages. These stages were the biological stages corresponding to maturation, the mean survival age, the $10 \%$ survival rate, and the highest age reported for an individual belonging to the respective species. The time intervals between the resulting intersections were normalized linearly for the mean survival age. Thus, the sampling time points examined in this study for a specific species (blue circles) matched the biological age category in all the other species. (B) Data sampling scheme and categorization of the high-throughput transcriptomic data according to age parameters: for each of the 4 species of interest, up to 4 tissue types were sampled at mature, aged and old-age stages, from which 3 comparisons were deduced to identify DEGs during early aging (mature vs. aged), late aging (mature vs. old-age), and longevity (aged vs. old-age). 
their lifespans to key biological stages: Time of maturity, average survival and extended survival reached by $10 \%$ of the individuals as well as the maximum reported age at death (see Figure 1A). According to these time scales, we defined 3 representative time categories comparable for each species - mature, aged, and old-age. Among these, we performed 3 different age comparisons denoted as early aging (mature vs. aged), late aging (mature vs. old-age), and longevity (aged vs. old-age) (see Figure 1B). Information on maturation and survival of the 4 species was underlying different sources: for $H$. sapiens [2224]; for M. musculus [25-28]; for D. rerio [29-32]; and for $N$. furzeri [19, 33-35].

The RNA-Seq libraries were filtered and qualitytrimmed with Prinseq (v0.20.3) [36], i.e., to achieve a minimum sequencing accuracy of $99.0 \%$, all reads were truncated at both ends until a Phred quality score of 20 or more was reached. Thereby, reads below a length of $15 \mathrm{nt}$ or those comprising at least 3 ambiguous $\mathrm{N}$ bases were removed. Read qualities were monitored by FastQC (v0.11.3; https://www.bioinformatics.babraham.ac.uk/projects/fas tqc/). TopHat2 (v2.1.1) [37] was used with default parameters to map the quality-trimmed RNA-Seq libraries to the corresponding reference genomes, thus, allowing spliced reads and single read mapping to multiple best-fitting locations. Featurecounts (v1.5.3) [38] was applied to perform read counting, with all reads being normalized to transcripts per million (TPM) [39] using the following formula:

$$
T P M_{i}=\frac{c_{i}}{l_{i}} \times\left(\frac{1}{\sum_{j \in N} \frac{c_{j}}{l_{j}}}\right) \times 10^{6}
$$

where $c_{i}$ indicates the raw read count of gene $i ; l_{i}$ represents the cumulative exon length of gene $i$; $N$ comprises the number of all genes in a given annotation. Genes with a TPM value $\leq 1$ in each sample were considered not to be expressed and were excluded in all subsequent expression analyses. The Bioconductor DESeq2 (v1.10.0) package [40] was utilized to identify DEGs in the 3 age comparisons of every species and tissue studied. Benjamini and Hochberg's False Discovery Rate (FDR) approach [41] was employed to adjust the calculated $p$-values for multiple testing. All genes with an adjusted $p$ value of less than 0.05 were assumed to be significantly differentially expressed. Details regarding DEG results, raw and normalized counts are accessible in the online supplement (https://osf.io/gtu2w/).

\section{Compilation of conserved $\mathrm{CR}$ genes and t-SNE dimension reduction}

We focused on the core genes that establish the circadian cycle and their direct regulators. Based on a comprehensive literature-based data curation, a list of 46 CR-related genes common in all 4 investigated species was compiled (for details see online supplement https://osf.io/ctv2r/). These 46 CR-related genes were further categorized into 4 groups: 9 CR core genes (CRCGs), 11 transcriptional regulators of the CRCGs, 9 post-transcriptional regulators of the CRCGs, and 17 post-translational regulators of the CRCGs. Figure 2 illustrates the specific interplay between the CRCGs and their different regulators. In order to assess the expression similarities of the RNASeq data related to these $46 \mathrm{CR}$-related genes, in terms of the parameters species, organ and age, the tdistributed stochastic neighbor embedding (t-SNE) algorithm [42] was applied for dimensionality reduction (see online supplement https://osf.io/e $7 \mathrm{k} 9 \mathrm{~g} /$ ).

\section{Age-dependent gene expression variances}

The coefficient of variation expressed as the ratio of the standard deviation and the mean of each CR-related gene was assessed to approximate the overall variance in gene expression among the reference time categories defined for the individual species and tissues. Variance differences among time categories were statistically assessed by two-tailed $t$-test and corrected for multiple testing by the Benjamini and Hochberg's False Discovery Rate (FDR) approach [41].

\section{Data availability statement}

The datasets analyzed for this study can be found in the NCBI's Gene Expression Omnibus database (https://www.ncbi.nlm.nih.gov/geo/): (Homo sapiens: GSE75337, GSE103232; Mus musculus: GSE75192, GSE78130; Danio rerio: GSE74244 and Nothobranchius furzeri: GSE52462, GSE66712).

\section{RESULTS AND DISCUSSION}

\section{Age-related effects on CR gene expression across species}

Vertebrate CR has been well characterized in mice and zebrafish, but less in other species. Moreover, to the best of our knowledge, a comprehensive comparison of age-dependent CR regulation between phylogenetically distant species is not yet available. This study compares 4 tissues (brain, blood, liver, and skin) of 4 vertebrate species (the 2 mammals, $H$. sapiens 
and $M$. musculus, and the 2 fish, $D$. rerio and $N$. furzeri) at 3 different ages.

\section{CR-related gene expression discriminates among species and tissues rather than age}

First, we evaluated the homogeneity of the analyzed transcriptomic data. Thus, we applied the t-SNE dimensionality reduction algorithm on the investigated RNA-Seq libraries based on the 46 selected CR-related genes (see online supplement https://osf.io/e7k9g/). Thereby, samples clustered primarily at the species' tissue level, i.e., no inter-species tissue cluster emerged. As the only exceptions, the 2 fish liver samples were merging and partially overlapped with the $N$. furzeri skin samples. Among all clusters, almost no agespecific segregation of either age category was discerned. This observation suggests that aging, from a global perspective, might have a relatively subtle impact on the global transcriptional level of CR-related genes, which seems to be a common finding shared by related studies addressing other target genes underlying the same dataset [43, 44]. However, a certain separation between the mature, aged, and old-age categories was observed for brain samples of $D$. rerio. For $N$. furzeri, only the old-age brain samples were distinct from the 2 younger time points. For $M$. musculus, no such separation was found. Additionally, the old-age skin samples of $N$. furzeri were distinguishable from the mature and aged samples. Human samples of either tissue were devoid of age-specific cluster formation.

\section{General observations of age-related effects on the CR regulation across species}

However, at the single-gene level, we found 42 out of the 46 CR-related genes to be differentially expressed in an age-dependent manner in at least one species and one

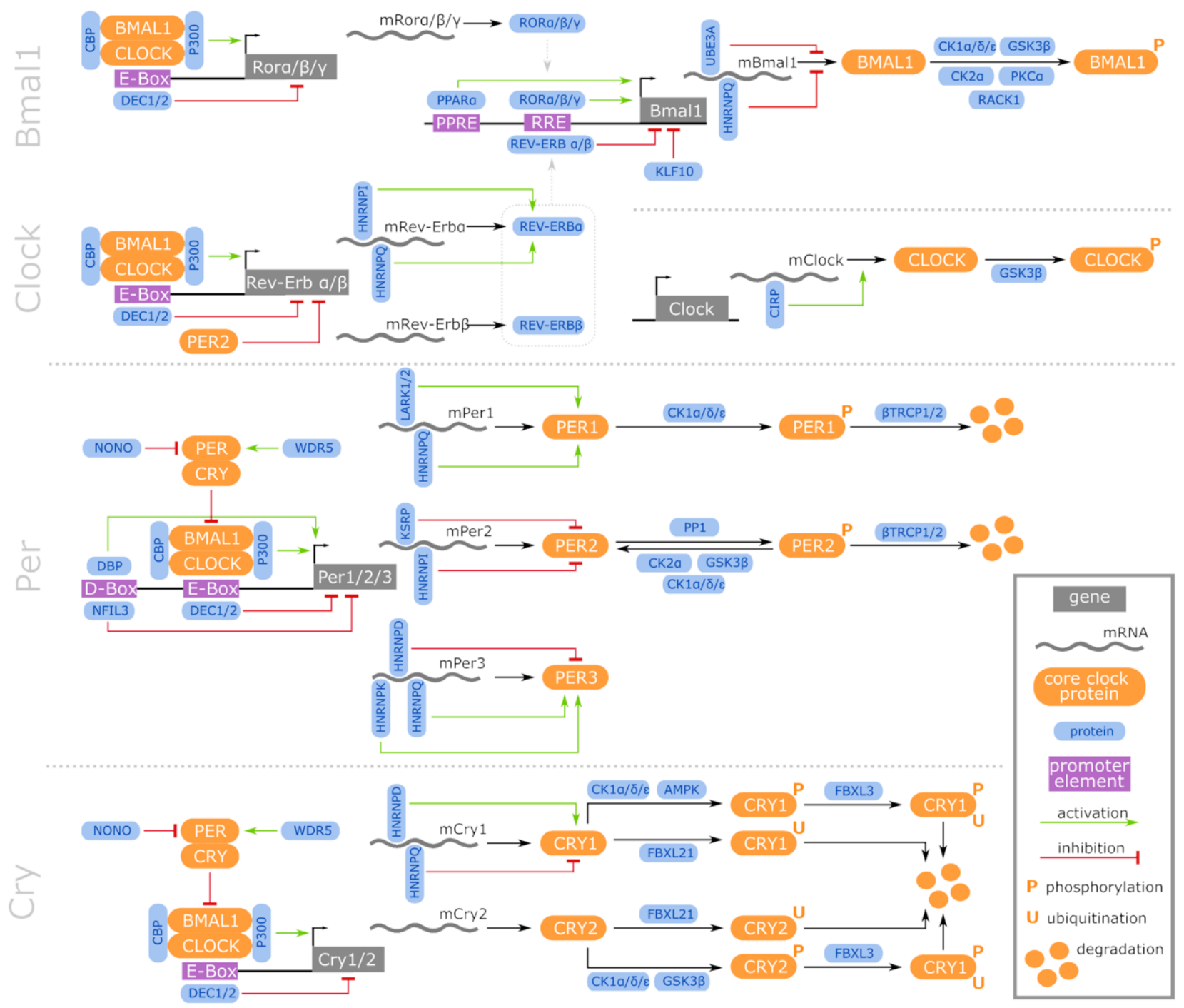

Figure 2. Regulatory network of vertebrate core CR genes, including their transcriptional, post-transcriptional, and posttranslational interaction partners that constitute activating and inhibitory feedback loops. Further information is accessible in the online supplement https://osf.io/ctv2r/. 
age comparison (see Figure 3). In total, out of these 42 DEGs, we identified 37 DEGs in D. rerio, 22 DEGs in $N$. furzeri, and 21 DEGs in M. musculus. In the human samples, we found 5 DEGs exclusively within the comparison between the mature and the old-age skin samples. Most of the DEGs identified belonged either to

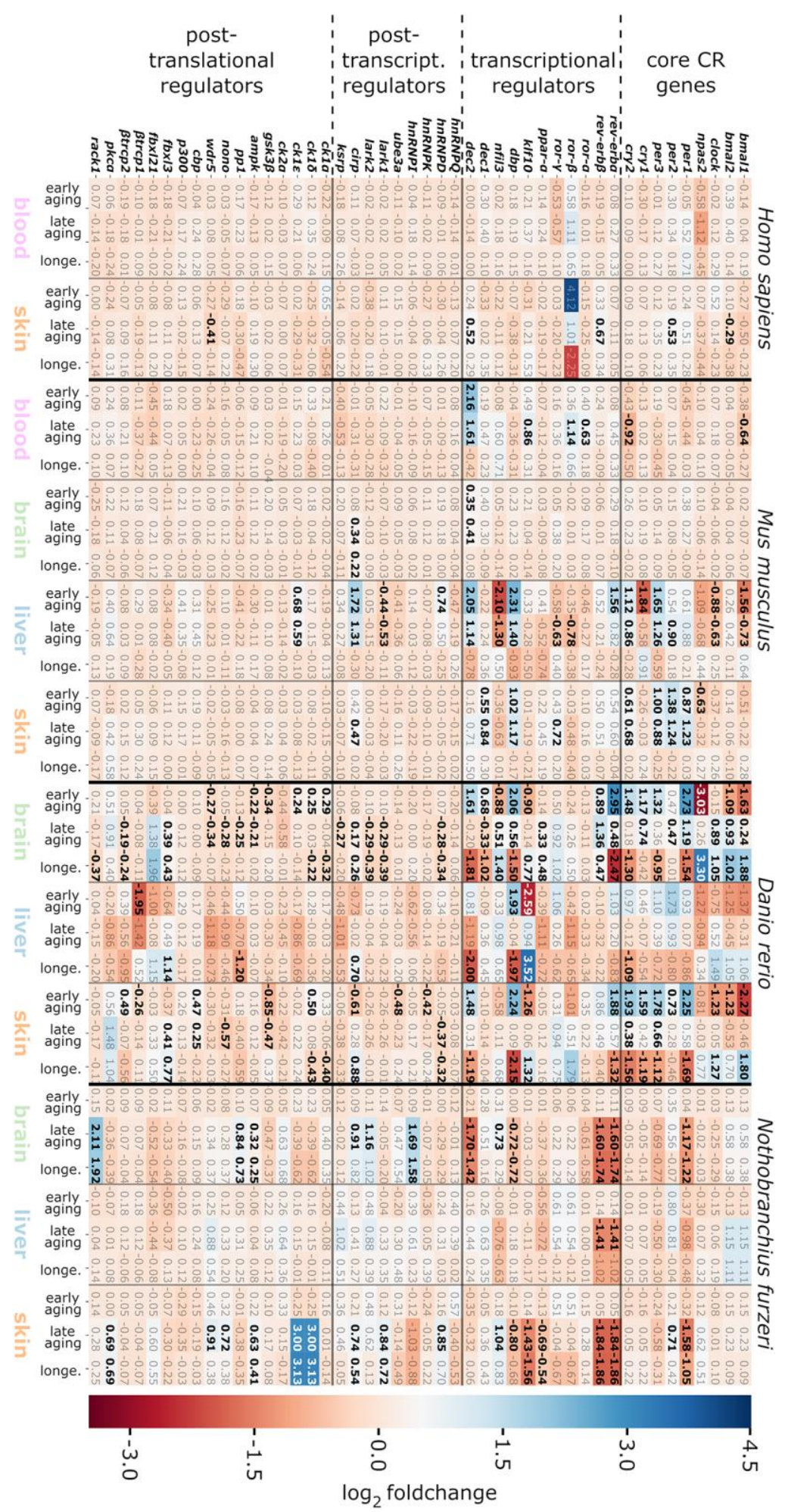

Figure 3. Heatmap representing $\log _{2}$ fold changes of the CR-related genes for the species, tissues and age categories investigated. DEG up-regulations in the course of aging are indicated by positive values displayed in blue, whereas down-regulations are shown by negative values in red. Significant gene expression alterations are highlighted in bold. The abbreviation longe. stands for the longevity age comparison (aged vs. old-age samples). For details, see the online supplement https://osf.io/9c3j4/. 
the $\mathrm{CR}$ core genes or to their direct transcriptional regulators.

A possible explanation for the low amount of DEGs identified in human samples might relate to the circumstance that the probands were not exposed to controlled environmental conditions and synchronization for day-night-cycle activity. Even though the human samples were collected during similar daytime, the variance in expression of individual genes was higher than within the other species. This higher intra-tissue gene expression variance in the human samples was also reflected by the broader sample distribution within the t-SNE projection. The age-related transcript changes represented by the 5 DEGs identified in the human samples, 2 of which belong to the $\mathrm{CR}$ core genes, might underestimate the impact of aging on human $\mathrm{CR}$ regulation.

Interestingly, represented by 16 DEGs, the most frequent transcript changes were found within liver samples of $M$. musculus aside from the DEGs manifesting in the skin, blood, and brain samples (9, 6, and 2 DEGs, respectively). Such observations are in accordance with the recent illustration that, at least in mouse, aging has a more profound impact on the liver than on other organs, in terms of gene expression levels, which might explain the pronounced number of DEGs within the mouse liver samples [44, 45]. This finding contrasted with the expression pattern discovered in both investigated fish species, where most of the DEGs arose from brain and skin specimens rather than from liver samples. A putative explanation might originate from the nonhierarchical and decentralized operation of the circadian system in fish [7]. Fish tissues are characterized by a direct light responsiveness and independent circadian pacemakers, which maintain the CR in different organs (for a recent and comprehensive review, see Steindal et al. [46]). Furthermore, since organs are differently affected by the sequela of aging, the peripheral representatives of the circadian system in fish might also respond individually with respect to their $\mathrm{CR}$-associated gene expression patterns. In terms of biological requirements, each tissue might adapt individually to age-related influences on its organ-specific pacemakers as autonomous from a central pacemaker.

In mouse, representing mammals, cirp was the only DEG identified within the longevity comparison. Cirp is a post-transcriptional regulator of the $\mathrm{CR}$ core gene clock and of the crucial transcriptional CR regulator $d b p$ [47]. In contrast, in $D$. rerio and $N$. furzeri, the comparison between aged and old-age samples, categorized as longevity, resulted in 25 DEGs and 16 DEGs, respectively, also including cirp. Such difference in $\mathrm{CR}$ regulation during longevity between mammals and fish might arise from a putatively earlier adaption to biological aging effects in the analyzed human and murine tissues or be due to the ability of fish to adjust their CR at higher ages.

In $N$. furzeri, the early aging comparison remained devoid of CR-related DEGs, which emerged only in comparison with the old-age samples. Therefore, the age-related changes in the $\mathrm{CR}$ regulation might have a positive effect on the longer lifespan of the old-age individuals. This explanation remains speculative, requiring further investigations of the $\mathrm{CR}$ in $N$. furzeri, similar to studies available in $D$. rerio $[5,48]$.

\section{Conserved expression patterns of per2 and dec2 during aging}

Out of the 42 DEGs, 2 were shared in all 4 investigated species in at least one tissue, 11 in 3 species, 15 in 2 species, and 14 DEGs were identified to be speciesspecific (see Figure 4). Among them, the core circadian system regulator per 2 and its competitive transcriptional repressor $d e c 2$ were differentially expressed during aging in all 4 investigated species.

Per2

Per2 is a member of the per family and plays a crucial role in CR maintenance as one of its core regulators. As part of the negative branch of the $\mathrm{CR}$ regulatory feedback loop system, Per2 forms a heterodimer with Per1 and Cry1/2, thereby suppressing the Bmal1:Clock complex and inhibiting its own expression [49, 50]. Deregulation of per2 is known to play a role in cancer risk and progression of several tumor types (for a brief review, see Wood et al. [51]). However, overexpression experiments correlated per 2 with a tumor-suppressing function [52-54]. In our data, we detected per 2 to be up-regulated with age in the skin of all 4 species analyzed, in the brain of $D$. rerio and in the liver of $M$. musculus. Such shared rise in its age-related expression suggests that prevention of tumor formation during aging might involve a conserved per2 function. Considering its dual role, as a potential tumor suppressor and as a core regulator of the CR, agerelated influences on per 2 regulation by other CRrelated genes, like bmall or clock, might potentiate its function in tumor suppression. Additionally, per 2 is described to stimulate autophagy levels in mammals [55]. Age-dependent decline in the efficiency of autophagocytic cascades is one of the main driving forces of aging [56] and restored autophagy was evidenced to prolong the lifespan of several organisms [57-59].

Dec2

Dec2 belongs to the basic helix-loop-helix family of transcription factors. It operates as a competitive 
transcriptional repressor of E-box-dependent per gene family transcription and thus plays a crucial role in executing the negative branch of the CR feedback loop as well as in CR downstream regulations [60, 61]. Across all age categories, we found $d e c 2$ to be differentially expressed in each of the 4 species including the 4 tissues investigated. Whereas its expression was increased with age in both mammals, it was mainly decreased in fish, with the exceptions of an inconsistent regulation in $D$. rerio brain and skin samples. There, dec 2 displayed an increased expression in the early aging comparison, followed by a decrease in its transcript level in the old-age as compared to the aged individuals. Dec2 is involved in several other aging-related cellular processes, like tumor formation [62], hypoxia [63], apoptosis [64], and inflammation $[65,66]$. Similar to per2, the expression of $\operatorname{dec} 2$ is highly dependent on the interaction with other CRrelated genes [61], making it prone for age-dependent deregulation of the CR. Moreover, dec2 is established for its oscillating expression in the SCN. Here, our data provide indications that $d e c 2$ expression and regulation is not restricted to the master CR pacemaker in the brain hypothalamus, but it is apparently also conserved in skin, liver, and blood. Whether dec2 transcripts, similarly to $d b p$, belong to the targets of the posttranscriptional regulator Cirp, particularly in old tissue, requires further exploration [47].

\section{Tissue-specific gene expression changes over the course of aging}

Although our set of target genes comprised 46 conserved CR-related regulators among the 4 investigated species, only a few of them showed a comparable expression pattern during aging, as discussed above. One reason is pulsed transcription of $\mathrm{CR}$ genes and the individual oscillatory responses to entrainment factors by different tissues [11, 67, 68]. Thus, concerning our analysis, we focused on agerelated inter-species $\mathrm{CR}$ gene expression patterns underlying the same tissue rather than comparing several tissues from an individual species.

\section{Brain}

Brain-mediated CR regulation, beyond a key pacemaker role of the SCN, has so far not been extensively studied in the context of aging $[69,70]$. Likewise, CR phase

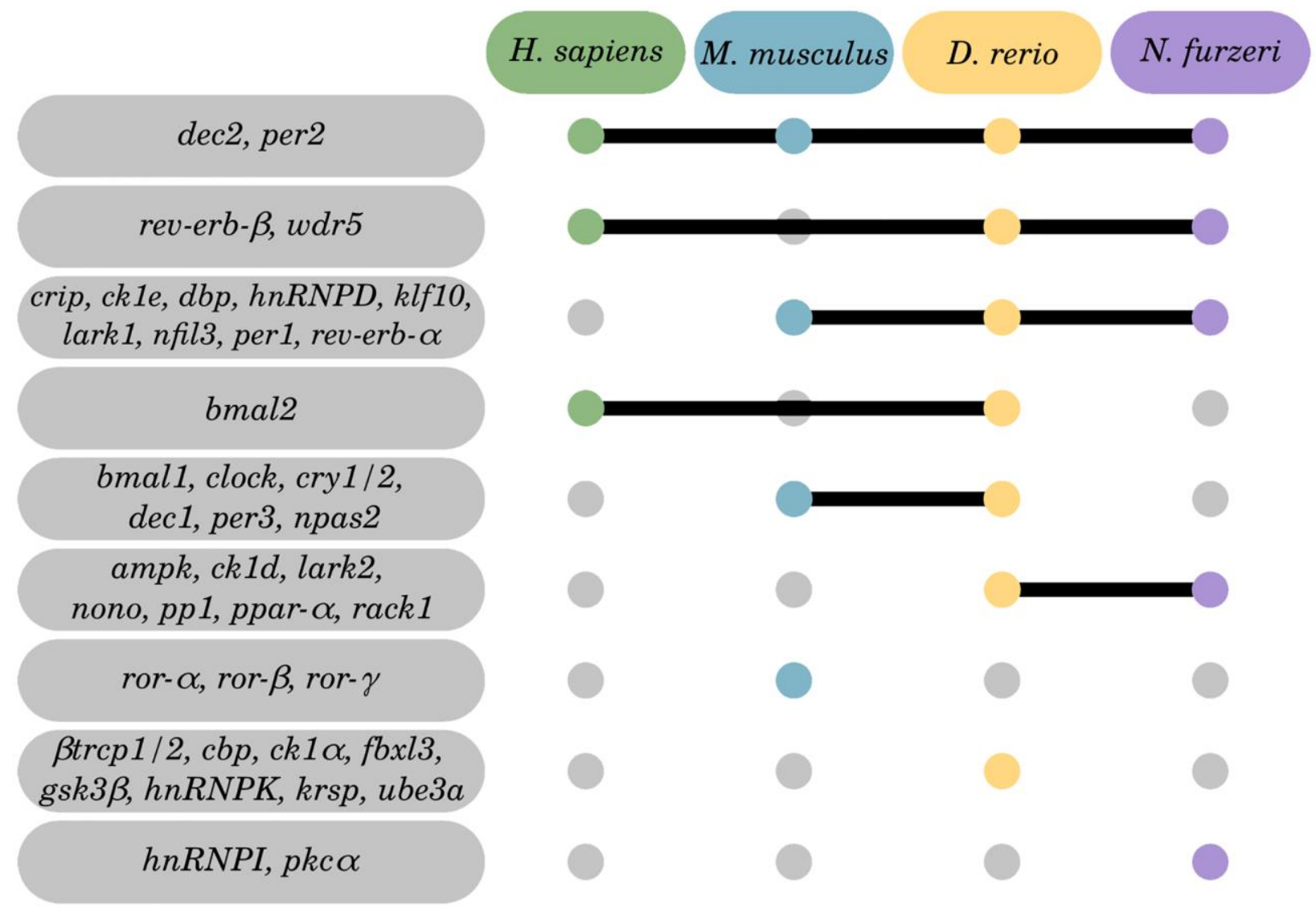

Figure 4. Inter-species overlap of CR-related genes regulated by aging. Of the 42 identified DEGs, 28 were identified within 2 or more species, and 14 DEGs to be species-specific. Tissue-specific Venn diagrams can be found in the online supplement: https://osf.io/3mgc6/. 
oscillations entrained by non-SCN brain tissue might be comparable to other non-SCN tissue as recently evidenced in the work by Yan and colleagues [68]. Also, deletion of CR key players in brain such as bmall/2 and clock in conjunction with npas 2 was identified to cause severe age-related astrogliosis [14]. By contrast, the deletion of bmall alone lead both to structural and molecular changes [14].

In this study, brain-specific regulations of the CRrelated transcriptome were characterized in mice and 2 fishes. As these brain analyses in mice and fish were based either on a hemisphere or a whole brain, respectively, our data are not specific for age-related changes eventuating in the hypothalamus. Age had almost no impact on the expression of CR genes in murine brain, independently of the age category addressed, suggesting a robust circadian system over mature lifetime. In contrast, CR-related genes were heterogeneously and strongly altered in both fish brains, thereby reaching $\log _{2}$ fold changes ranging from -3.03 to 3.30 in D. rerio and -1.74 to 2.11 in $N$. furzeri (see Figure 3). Thus, CR regulatory input from SCN-independent neuronal populations in fish brains (potentially in addition to the pineal complex and photoreceptors of the eye) might have stronger impact than in mammals or be subjected to pronounced agerelated perturbations in fish. In an earlier study by Zhdanova et al. [5], the CR core genes bmall and perl were described to be down-regulated in the pineal glands of aged zebrafish, but not clock. Here, we found an altered, diminished expression level of clock in the early aging comparison of the $D$. rerio brain samples as well. However, in the old-age $D$. rerio brain specimens the expression of these $3 \mathrm{CR}$ core genes was stimulated both in comparison to mature and aged samples. The detection of $\operatorname{dec} 2$ and cirp as the only DEGs that were altered both in the murine and fish brain samples might highlight their roles in the integration of peripheral CR oscillators, such as from liver and skin, into superordinate CR pulses.

\section{Cirp}

The highly conserved stress-inducible RNA-binding protein Cirp is known to directly interact and stabilize the mRNA transcripts of the CR core gene clock, enhancing its translation [47]. As cirp is also expressed in a broad tissue variety, the observed up-regulation of cirp in aging organs from mouse and both fishes might follow the biological purpose to counteract age-related $\mathrm{CR}$ alterations and stabilize the CR under increased cellular stress. Accordingly, cirp expression is responsive to hypoxia, UV radiation, body temperature, sleep patterns, metabolic cues, and DNA damaging stressors [71]. However, such role of cirp in the restitution of the $\mathrm{CR}$ robustness in the context of aging deserves further experimental proof.

Consistent with our evidence of a well-stabilized CR system in the brain, the resilience of the mammalian hypothalamus towards aging was recently demonstrated [72]. In this study by Eghlidi et al., underlying microarray and qRT-PCR techniques on young and old tissue specimens, the diurnal expression of SCN regulatory genes was unaffected by the age of the studied rhesus monkeys. Consequently, the authors concluded that $\mathrm{CR}$ misalignment in subordinate regulatory body systems might be the source of altered behavior in aged rhesus macaques. Moreover, our data indicate that, despite a decentralized CR regulation, $\mathrm{CR}$ core genes in fish are similarly conserved as in mammals, with SCN-paced hierarchical CR. Likewise, several fish orthologues of murine CR-interacting transcription factors represent targets of the CR core gene bmall [15].

\section{Blood}

Although longitudinal CR studies are frequently performed with blood, the data from the cross-sectional approach used in this study argue for a rather weak agerelated CR representation in this tissue. Likewise, no age-associated alterations of the CR-related transcriptome were detected in nucleated cells from human blood. In mice, only a few CR-related genes were identified to be differentially expressed, including representatives of the CR core gene category and some of their transcriptional regulators. The DEGs identified in murine blood displayed $\log _{2}$ fold changes between -0.92 and 2.16, with the strongest effects being found for cry2 ( $\log _{2}$ fold change: -0.92) and $\operatorname{dec} 2$ ( $\log _{2}$ fold change: 2.16). The Dec2 transcription factor sustains the negative arm of the circadian core feedback loop that suppresses the Bmal1: Clock protein dimer. Dec2 is involved in impaired sleep homeostasis and short sleep manifestation [73] and signals on pathways propagating tumor progression and growth [62], which, on the other hand, are altered by the aging process [74].

\section{Liver}

\section{Kfl10}

Liver represented the organ with the most frequent and most prominent CR-related gene expression alterations found in $M$. musculus and D. rerio, respectively, with the latter displaying $\log _{2}$ fold changes between -2.59 and 3.52 for the klf10 orthologue. Klf10 is a crucial repressor of bmall and was found down-regulated in the aged $D$. rerio liver samples compared to the mature and old-age samples, the 2 of which displayed similar expression levels. Reduced expression of bmall is known to contribute to aging, e.g., through the association with a higher abundance of reactive oxygen 
species, thereby propagating cellular senescence, at least in mice $[75,76]$.

\section{Nfil3}

In both the early aging and the late aging comparison in mice, we found $n f i l 3$ to be lower expressed ( $\log _{2}$ fold changes of -2.1 and -1.3 , respectively), suggesting that the amount of Nfil3 might be decreased in aged and, to a less extent, in old-age mice. Nfil3 binds to D-box elements residing in the $\mathrm{CR}$ core gene promoters, such as of the per family members perl/2/3 (see Figure 2). Nfil3 negatively regulates the transcription of these genes by competing with PAR-domain basic leucine zipper (PAR bZip) transcription factors such as Dbp, Hlf, and Tef, in an anti-phasic oscillatory manner [77]. In accordance with the suppressor function of Nfil3 on the per family, its observed down-regulation coincided with an up-regulated expression of per 2 and per 3 in the same age comparisons. Similarly, we found $d b p$ expression elevated in the corresponding age categories in mice, putatively entailing higher Dbp protein levels with age, again with a less pronounced regulation in the long-lived than in the aged individuals.

\section{Dbp}

Dbp is a D-box binding competitor of Nfil3, promoting the expression of per genes [78, 79]. The transcription of $d b p$ itself is suppressed by per gene family members [78]. The deregulation of per genes has been associated to tumor progression and a poor cancer prognosis [54, 80]. In addition, some studies found that overexpressed per 1 and per 2 can act as tumor suppressors in a timedependent manner in some malignancies [52-54]. In the $D$. rerio liver samples, the expression of $d b p$ was, similarly as observed for mouse samples, increased in the early aging comparison with a $\log _{2}$ fold change of 1.93. Its expression was decreased in the longevity comparison with an equally strong $\log _{2}$ fold change of -1.97 , i.e., levels of $d b p$ transcripts were only elevated within the aged but not within the old-age individuals as compared to the young mature individuals. Dbp acts as a transcription factor of per family genes, and also controls the expression of many metabolic and detoxifying enzymes within the liver [81]. A reduced expression of $d b p$, along with other PAR bZip transcription factors, is correlated with epilepsy and early aging symptoms in mice [81, 82].

\section{Skin}

CR-related gene alterations in skin were represented across all 4 species, thereby showing $\log _{2}$ fold changes between -2.27 (for bmall in D. rerio) and 3.13 (for ckl $\delta$ and $c k l \varepsilon$ in $N$. furzeri). The most prominent expression changes were detected in $D$. rerio, engaging significant changes in 24 out of the 46 target CR-related genes. A similar pattern was found in the skin of
$N$. furzeri featuring significant changes in 17 out of the 46 target CR-related genes. Regarding human and murine samples, the number of CR-related DEGs was less abundant (5 out of 46 in human skin; 9 out of 46 in mouse skin). Overall, 4 out of 46 genes were shared as DEGs within the skin samples of at least 3 species, including genes encoding for Per1 and Per 2 of the core clock regulators, as well as Dbp and the RNA binding protein Cirp (Figure 4).

For cirp, we observed a higher expression either within the aged or old-age skin samples of M. musculus and both fish, similar to its expression pattern in the brain samples. As aforementioned, Cirp interacts with the Per members and with Dbp by stabilizing their transcript and protein levels. Dbp belongs to the PAR bZip family of transcription factors, which can activate the Per1 promoter in cooperation with Clock/Bmal1, while Clock/Bmal1 stimulates the transcription of Dbp. By contrast, Dbp transcription is suppressed by Per members [78]. Thus, these data support the assumption that the post-transcriptional regulator Cirp may play a key conserved role in adjusting $\mathrm{CR}$ oscillations under aging conditions. Thereby, it may influence core clock members and associated transcriptional modulators such as Per1, Per2, and Dbp, thus enabling a reciprocal consolidation of $\mathrm{CR}$ circuits. In how far Cirp exerts such an assumed CR stabilizing function under aging conditions, particularly in the skin, has to be further analyzed.

When addressing the early aging category in $D$. rerio skin samples, the DEGs found to be up-regulated comprised representatives primarily of the negative branch of $\mathrm{CR}$ core regulatory genes. In contrast, transcript levels of core regulator genes of the positive branch were down-regulated. Strikingly, this regulation was reverted to the contrary pattern within the longevity comparison. Collectively, this finding implies that expression of CR core genes, except for per 3 and cry2, was only altered within the aged individuals but remained stable within the old-age compared to the younger mature individuals. Such observation suggests that the exceptionally long-lived animals might have peculiar molecular measures to stabilize their CR. A similar pattern was observed in the $D$. rerio brain samples. However, the altered CR core gene expression levels were increased in the old-age individuals when compared to the mature individuals. A similar complementary regulation between the early aging and the longevity comparisons was discerned for the transcriptional regulators $k l f 10, d b p$, and $d e c 2$.

By contrast, the killifish $N$. furzeri showed no agingrelated reversion in the expression of CR-related genes in none of the organs analyzed. The respective CR-related 
genes reflected either a consistent increase or decrease in expression with aging. Thus, concerning the execution of chronobiological studies, zebrafish might be favorable over $N$. furzeri to monitor temporal CR alterations.

In murine skin samples, we detected an augmented expression of all CR core genes of the negative CR feedback loop branch during aging, except for cryl, the expression of which remained stable. Additionally, the transcriptional regulators $r o r-\gamma, d b p$, decl, and the posttranscriptional regulator cirp were also higher expressed with age.

\section{Differences in the expression stability of aged and long-lived individuals}

Aging is suspected to be responsible for disrupting the circadian system's regulation on both the master clock regulator level and the peripheral oscillators [4, 6]. Moreover, aging might be associated with an altered
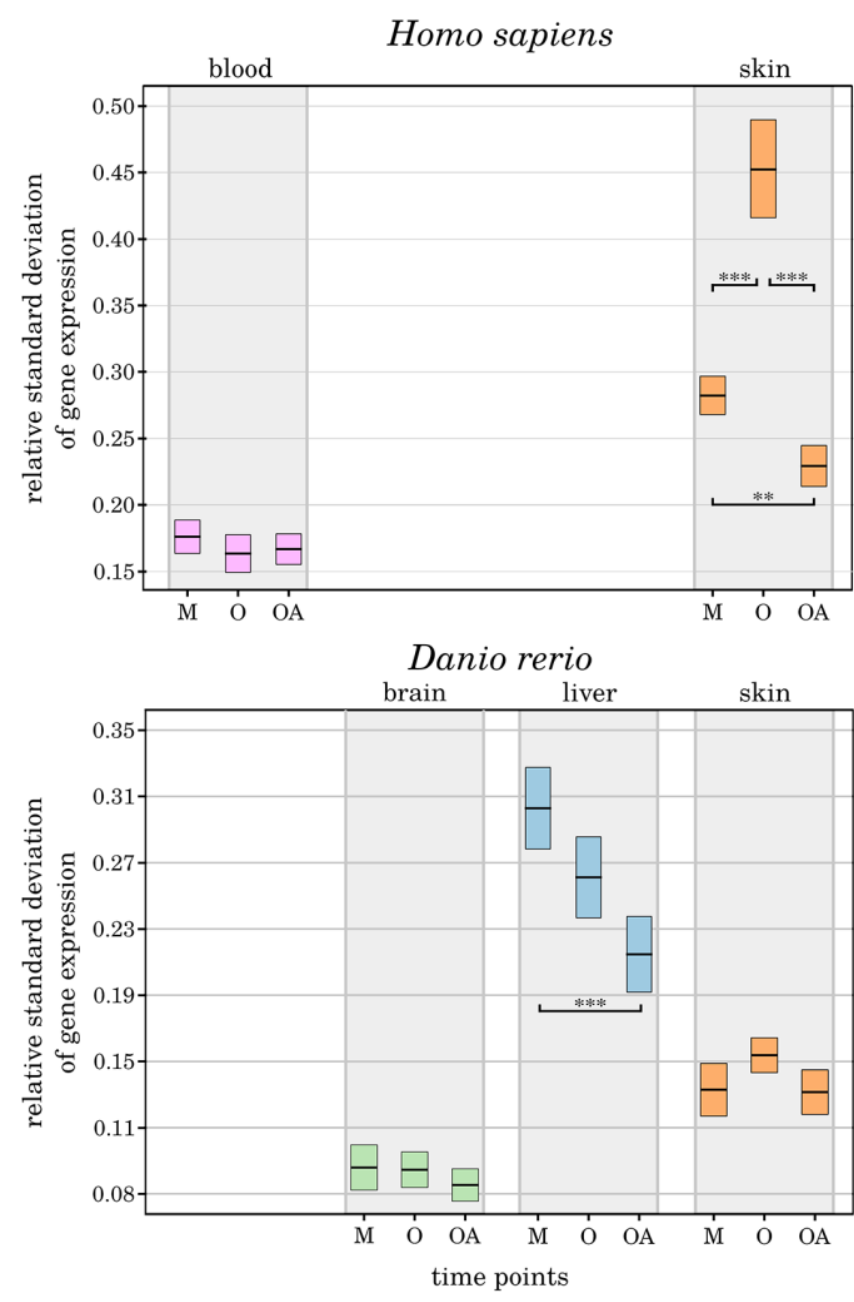

scatter of gene expression variances and serve as an indirect biostatistical indicator of organ and tissue aging [43]. Therefore, average changes in gene expression variances were specified for the 3 age comparisons delineated and applied as measure for the robustness of CR-related gene expression (Figure 5). In our previous study, we found genetic marker genes of senescence and inflammation to exhibit lower standard deviations in the old-age samples, indicating a more robust expression of those genes in the long-lived individuals [43]. However, for the CR-related genes a corresponding pattern was not discriminated. In 7 out of 16 species-related tissues within 4 tissue types, we detected no or only minor differences in the relative standard deviations between the age groups. The remaining tissues displayed heterogeneous changes between the respective ages, without the perception of a consistent pattern.

While human and mouse samples shared an overall increased variance in CR-related gene expression in the

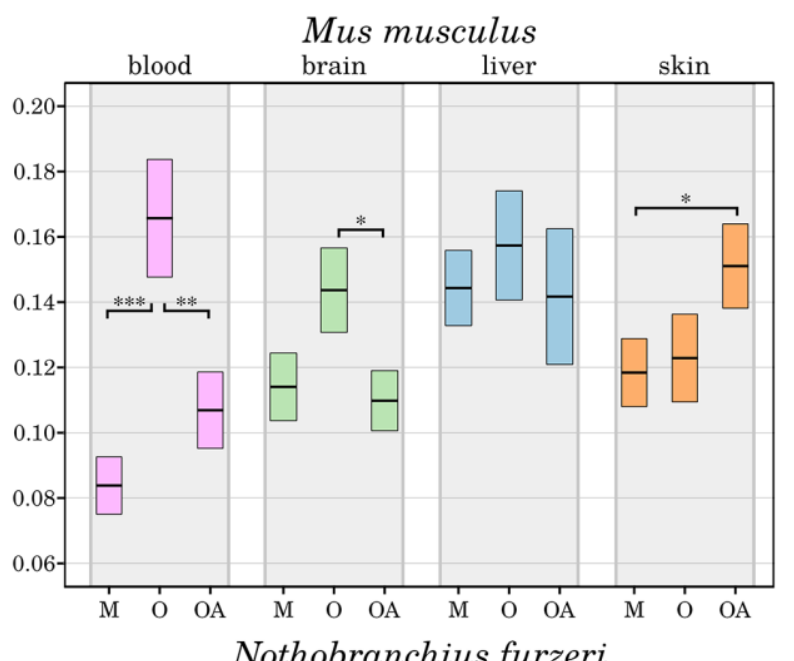

Figure 5. Age-dependent alterations in the robustness of CR-related gene expression. For the 3 age comparisons, different tissues, and species, the measured variance in the expression of CR-related genes is displayed as change in standard deviation. The extrema of the box plots represent the respective $2.5 \%$ percentiles. Age groups ( $\mathrm{M}$ - mature, $\mathrm{A}$ - aged, OA - old-age) are compared for their statistical significance: ${ }^{*} p$-value $\leq 0.01,{ }^{* *} p$-value $\leq 0.001,{ }^{* * *} p$-value $\leq 0.0001$. Details can be found in the online supplement: https://osf.io/g9uqz/. 
aged individuals, the long-lived individuals exhibited an unchanged or even lower expression variance than the younger mature individuals. The only exceptions were the human blood samples, without substantial agerelated impact on the expression variances. The mouse skin samples showed an increase in gene expression variance towards the old-age time point.

In $D$. rerio, the inter-group variability in gene expression was constantly decreasing with age selectively in liver samples. Exclusively in $N$. furzeri, the expression variances reflected a consistent pattern of an increased relative standard deviation towards oldage, pointing to a less robust expression of the CR. This observation supports our finding that DEGs were primarily arising from the old-age category in $N$. furzeri. Longitudinal studies are required to characterize the robustness of CR-related gene expression patterns within the different tissues and species.

\section{Cross-over mapping with established $\mathrm{CR}$ regulomes}

The primary shortcoming of our current study is rooted in the single time point-sampling of tissues and species included and the deficiency of a DEG expression normalization to a zeitgeber time (ZT) and inter-species alignment to rhythmic CR gene expression. To validate, in consideration of this caveat, the assignment of our underlying data to a chronobiological context, the set of CR-related DEGs exhibiting age-related alterations were aligned to input data [83] as well as enriched output data of a newly established, refined highconfidence network of CR-regulated genes in humans, called NCRG. This reference network was retrieved by a bioinformatical meta-analysis of multi-modular highthroughput CR-related data [84].

The underlying input inter-connectome of a core clock $(\mathrm{CCN})$ and an extended core clock network (ECCN) implementing update connections according to PubMed-available data comprised 43 CR-related elements displaying more than 200 regulatory interdependencies [83, 84]. First, when mapping our 46 CR-related genes (Figure 3), 42 out of which proved to be differentially expressed during the aging process, we identified that all of the $14 \mathrm{CR}$ genes nominated to be engaged in the $\mathrm{CCN}$ in humans [84] were represented in the category of core clock regulators and immediate transcriptional regulators in our inter-species study. This $100 \%$ overlap covered both the positive and negative branches of the circadian feedback loop (Figure 6). Second, as for the ECCN, 19 out of 29 (65.5\%) CR-related elements designated to belong to the human ECCN [84] overlapped with the DEGs nominated as $\mathrm{CCN}$ interaction partners in our interspecies approach (Figure 6). Third, among the 118 genes, newly nominated by Lehmann and colleagues to be potential ECCN targets and thus becoming part of their innovative, most comprehensive NCRG in humans, we found 2 of them, i.e., Csnklal and $H n R N P I$, to overlap with our DEGs output. As the reference network established by Lehmann and colleagues is not tissue-specific, we could not match for inter-tissue peculiarities.

Considering such correspondence, this large-scale curated human CR-regulatory network might be well complemented by the human homologs of the 13 additional genes we de novo assigned to the $\mathrm{CR}$ regulome in our non-synchronized but inter-species and tissue-specific approach, 11 of which represented DEGs with a significant expression modulation by aging (Figure 6). These additional genes encompass one transcriptional and numerous post-transcriptional and post-translational regulators. In support of such notion, only 19 out of the 118 genes newly assigned to the expanded NCRG network suggested by Lehmann and colleagues showed circadian expression patterns in mice [86]. Thus, mutual overlap and uniqueness of DEGs in the inter-species comparison might broaden our evolutionary view on candidate genes relevant in hierarchical CR regulation, development of CR-related disorders in humans, and chronotherapy.

Among these additional 11 CR-related DEGs, the structurally conserved gene encoding for the Klf10 zinc finger transcription factor of the Krüppel-like family was found engaged in the class of transcriptional regulators. Klf 10 functions as an effector of the TGF- $\beta$ signaling pathway. It operates as a repressor of cell proliferation and inducer of apoptosis and thus is assumed to play a role in cancer growth and progression. Moreover, Klf10 is indicated to propagate cardiovascular diseases, diabetes, and cancer by activating pro-inflammatory tissue responses [85].

The DEGs assigned to the category of posttranscriptional regulators included several representatives of the HnRNP class of RNA interacting factors (HnRNP D, Q, I, K; HnRNPA18/Cirp), a large, conserved family of RNA binding proteins [86]. HnRNPs assemble on newly generated heterogeneous nuclear transcripts (hnRNA) to control their conversion into mature mRNAs. Along with this, they are involved in (pre-) mRNA processing, trafficking, and splicing. By transcriptome diversification, alternative splicing holds an established role in the adaptive stress response activated during aging. Accordingly, deregulation of the spliceosome has been identified as a hallmark of aging, as it is associated with aging phenotypes and the expression of aging biomarkers in humans and agerelated disorders $[87,88]$. In terms of transcript affinity, 
many of the HnRNPs have oncogenes as direct targets as well as factors crucial in age-related neurodegenerative disorders, such as TERT/TERC, $K R A S, B R C A$, and APP mRNAs [88].

Similarly, the gene encoding the RNA-binding Khomology splicing regulatory protein Ksrp identified among these $11 \mathrm{CR}$-related DEGs, has a key function in regulating mRNA stability of genes involved in immune and inflammatory responses. As a member of the AUrich element-binding proteins (ARE-BP), it controls gene expression by targeted decay of mRNAs [89]. Ksrp prevents excess and harmful cytokine activation as a dominant target, e.g., after infection, in chronic neurodegenerative disorders, and cancer [90]. Repression of Ksrp target genes has been discussed to serve as a conserved anti-inflammatory mechanism across different species, including humans, mice, and rats, and across different tissues and cell types [91].

Further, ubiquitin E3 ligases, such as the product translated from Ube $3 A$ mRNA we detected among these DEGs, have been associated with several age-related neurodegenerative disorders. Ube $3 A$ is involved in genomic imprinting of experience-dependent ocular plasticity during development, and loss-of-function

\section{ECCN - Aging - interspecies}

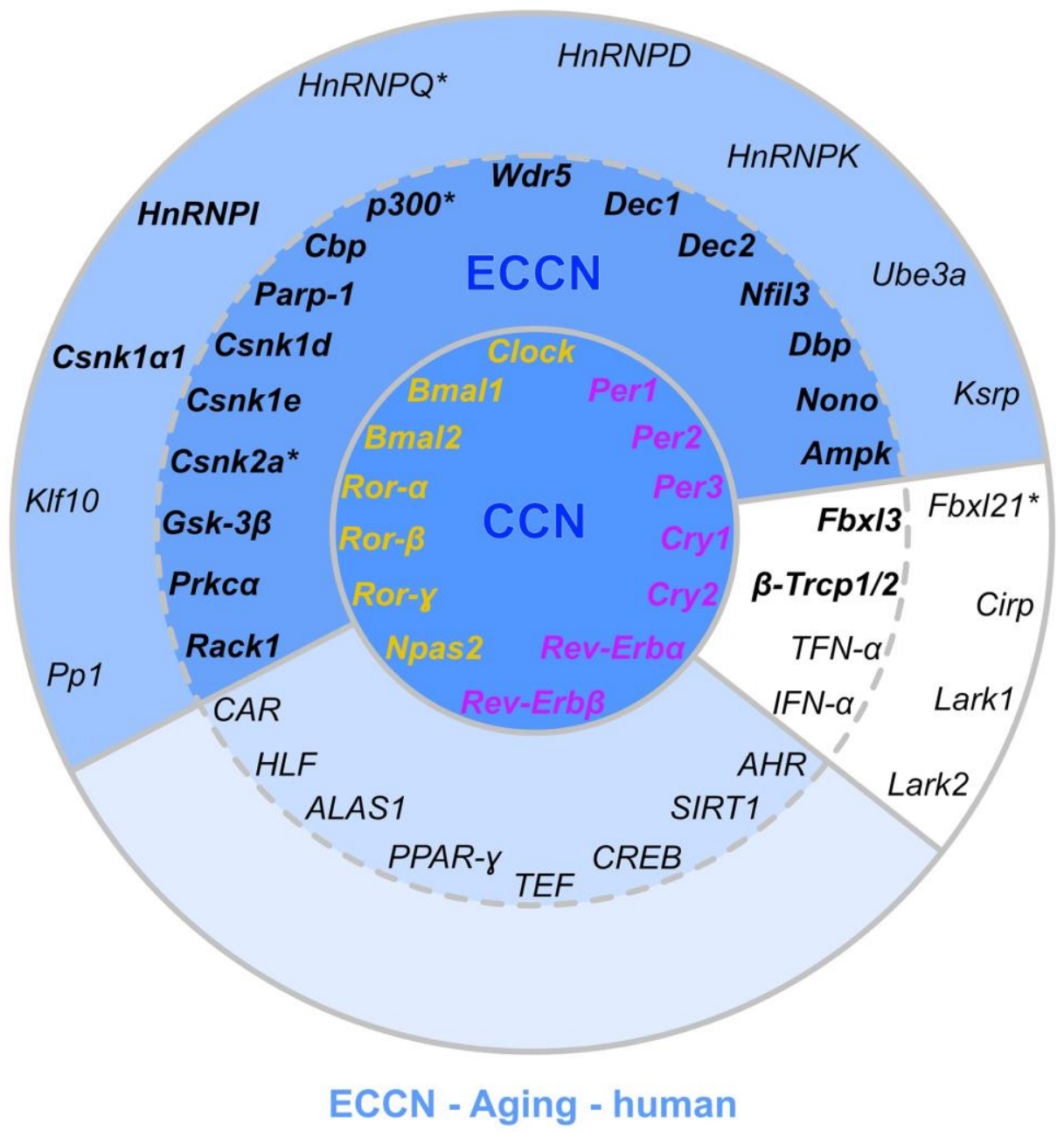

Figure 6. Matching of our CR-related DEGs evidenced to be regulated with aging with a curated human CR network. The core clock network (CCN; inner ring) is comprised of a positive (orange) and negative (purple) branch of core clock regulators and has been expanded to an extended core clock network (ECCN; middle ring) as determined by chronobiological meta-analyses in humans [84]. The majority of CR-related genes identified in the present study overlaps (represented in bold) with the human CCN ( $100 \%$ overlap) and ECCN (65.5\% overlap). The ECCN was complemented by 13 CR-related genes defined by our aging-related interspecies approach (outer ring), two of which overlapped with newly designated CR genes (bold in outer ring [84]). By mapping to a curated biomedical aging database [101], $85 \%$ of the presented interspecies (dark blue background) or human (light blue background) CR-related genes were identified to be agingassociated. The remaining factors (white background) have not yet been annotated to aging but might represent new candidates. ${ }^{*}$, curated CR-related genes without age-related differential expression within the interspecies comparison. 
models exhibit synaptic rigidity typical of the aged and neurodegenerative brain $[92,93]$. Ube 3 A protein levels have been shown to decline by $50-80 \%$ with aging in the mammalian cortex, as illustrated for humans, macaque monkeys, and cats, suggesting that this loss in Ube3A contributes to impaired cortical functionality and synaptic plasticity at higher ages [94].

Likewise, we found lark1 and lark2 to be among these 11 DEGs emerging in the inter-species approach. Products of the RNA binding lark genes have recently been identified to stabilize G-quadruplex structures in vertebrates and invertebrates in a conserved process [95] thus enhancing the transcription of target genes. As a primary target, Lark activates the expression of perl, thereby fundamentally regulating the length of $\mathrm{CR}$ periods [96]. Notably, lark transcript levels are not subjected to rhythmic pulses, whereas protein levels oscillate synchronously with Per1 in mice [96]. Such characteristics make lark an interesting novel candidate in understanding altered CR periodicity observed with aging.

Isoforms of the CsnK family (e.g., $\alpha, \beta, \gamma, \delta, \varepsilon)$ and their splice variants have a vital role in the phosphorylation of molecules regulatory in transcription, translation, receptor-coupled signaling, apoptosis, cell cycle control, DNA repair, and chronobiology [97]. Further, they operate in multiple oncogenic signaling pathways [97]. Notably, the regulated CsnK2 $\alpha$ is discovered to phosphorylate Sirtuin 6 [98], which also operates as a longevity factor in mice.

Moreover, the post-translational $\mathrm{CR}$ regulator $\mathrm{Pp} 1$ has a function in the control of memory-related miRNAs. The activity of the Pp1 protein phosphatase is altered with aging, suggesting an implication in the manifestation of age-related cognitive impairments and dementia syndromes associated with progressive neurodegenerative disorders [99].

Further, the post-translational fbxl gene family, including the nominated $f b x l 21$ gene, is involved in cellular processes critical in aging and age-related pathologies such as cell cycle control and DNA damage response. The encoded F-box proteins represent E3 ubiquitin ligases that recruit their substrates and target them for degradation via interaction with specific motifs, or degrons, primed by post-translational modifications [100]. Deregulations are best characterized for cancer entities but suggest participation in pathologies implicating genomic stress conditions, including the aging process.

For additional support of the biomedical interpretation that our output DEGs are relevant for the aging process, we correlated our $46 \mathrm{CR}$-related genes with the entirety of genes currently annotated for a relevant function in aging. For this purpose, we utilized a newly established integrated database collecting and updating unprecedented multi-scale and in-depth knowledge on the biology of aging [101]. Notably, this publicly accessible biomedical database curates multi-omics information on the process of aging across different model organisms and species (https://bigd.big.ac.cn/aging/index). When applying the RNA-Seq database within the catalog of available data collections, we show that 39 out of 42 DEGs and 46 annotated genes summarized in our heatmap $(85 \%)$ are linked to the aging process. The high overlap of our results with pre-established bioinformatic CR pipelines and age-related functional -omic scale databases, as illustrated in Figure 6, supports the strength of our data despite a single time point study design.

\section{CONCLUSION}

Multiple efforts have aimed at mapping physiological and pathological processes to the circadian system either in individual $[12,102]$ or multiple organs [11] of a single species or in selected organs underlying a pathophysiological stressor [103]. However, the role of highly conserved $\mathrm{CR}$ regulation in physiological aging over time and across multiple species has not yet been addressed. Here, we used RNA-Seq data to profile the regulation of CR-related genes of 4 different species in a cross-sectional study in individuals ranging from young mature to old-age categories. We compared changes of the $\mathrm{CR}$ transcriptome in different organs within these species, including brain, blood, liver, and skin. In summary, our results show that modulations in CR-related gene transcription throughout aging are a conserved trait that is traceable across evolutionarily diverse species, ranging from humans to mice and fish. Thereby, we identified 2 CR-related genes, i.e., dec2 and per 2 , to be altered in all 4 species primarily in early aging and late aging rather than longevity, and 4 genes (cirp, klf10, nfil3, and $d b p$ ) with conserved agingrelated expression patterns in several organs and species.

In contrast to the analysis of CR-related mRNAs from different tissues, as studied, e.g., in zebrafish larvae [15], we realized an inter-organ comparison. In complementation to the work by Yan et al. (2008), who surveyed 14 tissues exclusively from mammalian species (i.e., human, monkey, and mouse) from a tissue gene expression atlas [68], we expanded the analyses of inter-organ relationships to 4 evolutionarily separated species and specified the regulation of CR-related genes in the context of aging. In comparison, the study by Yan and colleagues compiled microarray data from several 
previous studies and extracted 41 common CR genes from a total of 9,995 genes that oscillated in at least 8 out of 14 tissues in mice [68].

In our study, among the 4 species investigated, $H$. sapiens showed less regulation in terms of both the number and absolute expression changes of DEGs irrespective of the reduced availability of only 2 out of 4 tissue locations analyzed. If DEGs were identified, they belonged, except for $w d r 5$, exclusively to the core clock genes or the category of immediate core clock transcriptional regulators. $M$. musculus exhibited a similar hierarchical pattern of age-dependent CR regulation in terms of the 4 categories of gene regulators defined, concluding that primarily core $\mathrm{CR}$ genes and their transcriptional regulators were among the DEGs identified. In murine tissues, this pattern was best reflected by liver and skin. Such preponderance in the executive level of operating genes and their expression alterations was also present in $D$. rerio, with the strongest age-associated regulations found for core $\mathrm{CR}$ genes and transcriptional regulators. However, in contrast to mouse and human, D. rerio also showed broad age-related changes in the CR transcriptome at the level of post-transcriptional and post-translational regulators. In general, D. rerio exhibited the most versatile $\mathrm{CR}$ gene modulations concerning DEG numbers, $\log _{2}$ assessed gene expression alterations, tissues being involved in $\mathrm{CR}$ regulation, and gene regulatory categories covered. Thereby, the most prominent changes were attributable to brain tissue followed by skin. Unexpectedly, the short-lived killifish $N$. furzeri, which serves as an established aging model, was devoid of a corresponding CR regulatory pattern. Strikingly, we found almost no regulation of the core clock genes except for per family members. In general, the number of age-related CR genes in $N$. furzeri was much lower than in $D$. rerio and consistently represented by the gene regulatory categories downstream of the core CR genes. Accordingly, in contrast to $D$. rerio, the strongest DEG regulation manifested within post-translational regulators instead of core CR genes. Unlike M. musculus, both fishes showed a more complex involvement of brain and skin instead of liver in this aging-related CR pattern. In conclusion, $D$. rerio appeared to be best equipped to respond to aging-related CR-alterations in terms of transcriptome adaptions among the 4 species analyzed.

In general, we identified alterations in the $\mathrm{CR}$ regulation as an early characteristic of aging, in M. musculus and $D$. rerio, which emerged at or persisted into late aging in human, mouse, and fish, and further extended into longevity in $D$. rerio and $N$. furzeri. Though manifesting already during early aging, the inter-tissue and inter-species profile of DEGs engaged in CR regulation appeared broadest during late aging but less diverse at the old-age time points and best represented in their complexity by brain and skin. Thus, apart from a master role of the brain SCN in CR control, our data suggest an essential contribution of the skin to the transmission of chronobiological cues, as we detected a considerable amount of DEGs in the skin across all 4 species, possibly explained by the direct light exposure of this organ. Notably, in the 2 fishes, DEG patterns in the skin were comparable to that in brain tissue. In mice, age-related changes in CR-regulatory transcript levels were evident in the skin but not represented by brain tissue. These findings suggest that even in species relying on a primarily hierarchical CR orchestration, as found in mice and humans, peripheral subordinate organs such as the skin might compete with hypothalamic brain structures and contribute to CRrelated gene expression alterations during aging.

In support, expression of repressive core clock per 2 in the skin was increased during aging in all 4 species investigated. Age-related alterations of CR-related gene expression in light-sensitive skin suggest multi-level changes in the stimulus-dependent responsiveness of the $\mathrm{CR}$ regulatory system. Importantly, the regulation of per2 is mediated by complex feedback loops directly involving the cis-regulatory D-box enhancer element, which is recognized by PAR bZip and related transcription factors such as Hlf, Tef, CR activating $\mathrm{Dbp}$, and repressive Nfil3. As aforementioned, apart from per 2 itself, $d b p$ and $n f i l 3$ were found differentially expressed by age in mice and the 2 fish species, although D-box regulation differs fundamentally between mammals and fish (see online supplement https://osf.io/zc8d3/). Whereas in mammals, stimulation of D-box-activating transcription factors is indirectly regulated by interlocking feedback loops encompassing the CR output targets Bmal1 and Clock, D-box-binding transcription factors, such as Tef, are directly inducible by light in fish, conveying light-entrained regulation of clock genes [104]. Along with visible light, also UV light and ROS levels are engaged in D-box-driven, light-responsive gene regulation in fish but not mammals, targeting clock and DNA repair genes such as $d d b 2$ relevant in nucleotide excision repair (NER) and other stress response pathways [105, 106, 107]. Similarly, ROS levels are critically elevated during aging. In conclusion, we identified $3 \mathrm{CR}$ genes, i.e., per2, $d b p$, and $n f i l 3$, as being differentially regulated with aging, which are involved in direct light-induced gene expression in fish while representing clock output elements in mice and humans. The strong light responsiveness of D-box elements in fish might explain the pronounced expression alterations detected for $d b p$ and $n f i l 3$ in the skin of fish, while in the mouse samples, D-box regulation was dominant in the liver. 
Apart, the diametrical expression of $d b p$ and $n f i l 3$ illustrated in our heatmap for the murine liver is supported by other studies. Likewise, Ueda and colleagues identified an anti-phasic expression of these 2 transcription factors in frame of transcriptional circuit analyses established from in vivo liver and vectortransduced cellular models [79].

Additionally, we identified 6 genes in several tissues of at least 3 out of the 4 species investigated with an apparently conserved age-associated regulation. These genes act at each of the regulation levels established for the CR (core gene, transcriptionally, posttranscriptionally, and post-translationally) and have known biological roles outside the CR. Many of these functions are related to aging-associated diseases or driving factors, such as autophagy, hypoxia, apoptosis regulation, and inflammation. Thus, these genes might represent a conserved link between the circadian system and aging. However, since preliminary due to the nonsynchronized cross-sectional approach, our study deserves future replication in an additional dataset based on a longitudinal study design, including tissue synchronizations across the species of interest and the consideration of anatomic sub-regions when brain tissue is analyzed.

In how far CR regulation is causal to or a consequence of the aging process still remains to be explored. This work extends recent studies and yields a comprehensive new dataset linking $\mathrm{CR}$ factors to physiological aging across evolutionarily distinct species. It will support to dissect the alterations in chronobiology associated to latent or manifest disease conditions from $\mathrm{CR}$ characteristics in healthy aging and contribute to the identification of interventions that can improve the well-being of the elderly and extend a healthy lifespan.

\section{AUTHOR CONTRIBUTIONS}

Conceptualization: EB, AS, AK, MM. Data curation: EB, AS. Formal analysis: EB, AS. Data interpretation: EB, AS, DW, AK. Methodology: EB. Resources: HA, OW, MM. Validation: EB, AS. Visualization: EB, DW, AK. Writing - Original Draft Preparation: EB, AS, DW, AK, MS, MM. Writing - Review Editing: EB, AS, DW, AK, MM, OW. Project Administration: OW, MM. Supervision: MM. Funding acquisition: OW, AK, MM.

\section{ACKNOWLEDGMENTS}

We thank Maria Mittag for helpful discussion on circadian rhythm cascades. We are grateful to Ulrike Teschner for her assistance with human sample recruitment.

\section{CONFLICTS OF INTEREST}

The authors declare no conflicts of interest related to this study.

\section{FUNDING}

This work was supported by the "Else-KronerFresenius-Stiftung' (EKFS), the Deutsche Forschungs-" gemeinschaft (DFG, German Research FoundationClinician Scientist-Program OrganAge funding number WI 830/12-1; DFG, German Research Foundation funding number MA5082/15-1 to MM), the Ministry for Economics, Sciences and Digital Society of Thuringia (TMWWDG), under the framework of the ProExcellence Initiative RegenerAging (RegenerAging52-5581-413-FSU-I-03/14 to AK; RegenerAging-525581-413-FSU-I-03/15 to MM), under the framework of the Landesprogramm ProDigital (DigLeben-5575/109 to $\mathrm{MM}$ ), and the Interdisciplinary Center for Clinical Research of the Medical Faculty Jena (FF01 to AK; MSP09 to DW).

\section{REFERENCES}

1. Pilorz V, Helfrich-Förster $\mathrm{C}$, Oster $\mathrm{H}$. The role of the circadian clock system in physiology. Pflugers Arch. 2018; 470:227-39. https://doi.org/10.1007/s00424-017-2103-y PMID:29302752

2. López-Otín C, Blasco MA, Partridge L, Serrano M, Kroemer G. The hallmarks of aging. Cell. 2013; 153:1194-217. https://doi.org/10.1016/i.cell.2013.05.039 PMID:23746838

3. Pitt JN, Kaeberlein M. Why is aging conserved and what can we do about it? PLoS Biol. 2015; 13:e1002131.

https://doi.org/10.1371/iournal.pbio.1002131 PMID:25923592

4. Hofman MA, Swaab DF. Living by the clock: the circadian pacemaker in older people. Ageing Res Rev. 2006; 5:33-51.

https://doi.org/10.1016/j.arr.2005.07.001 PMID:16126012

5. Zhdanova IV, Yu L, Lopez-Patino M, Shang E, Kishi S, Guelin E. Aging of the circadian system in zebrafish and the effects of melatonin on sleep and cognitive performance. Brain Res Bull. 2008; 75:433-41. https://doi.org/10.1016/i.brainresbull.2007.10.053 PMID:18331912

6. Kondratov RV. A role of the circadian system and circadian proteins in aging. Ageing Res Rev. 2007; 6:12-27. 
https://doi.org/10.1016/i.arr.2007.02.003 PMID:17369106

7. Isorna $\mathrm{E}$, de Pedro $\mathrm{N}$, Valenciano Al, Alonso-Gómez ÁL, Delgado MJ. Interplay between the endocrine and circadian systems in fishes. J Endocrinol. 2017; 232:R141-59.

https://doi.org/10.1530/JOE-16-0330

PMID: $\underline{27999088}$

8. Hu K, Van Someren EJ, Shea SA, Scheer FA. Reduction of scale invariance of activity fluctuations with aging and Alzheimer's disease: Involvement of the circadian pacemaker. Proc Natl Acad Sci U S A. 2009; 106:2490-4.

https://doi.org/10.1073/pnas.0806087106 PMID: 19202078

9. Witting W, Kwa IH, Eikelenboom P, Mirmiran M, Swaab DF. Alterations in the circadian rest-activity rhythm in aging and Alzheimer's disease. Biol Psychiatry. 1990; 27:563-72.

https://doi.org/10.1016/0006-3223(90)90523-5 PMID:2322616

10. Farajnia S, Michel S, Deboer $T$, vanderLeest $H T$, Houben T, Rohling JH, Ramkisoensing A, Yasenkov R, Meijer JH. Evidence for neuronal desynchrony in the aged suprachiasmatic nucleus clock. J Neurosci. 2012; 32:5891-9.

https://doi.org/10.1523/JNEUROSCI.0469-12.2012 PMID:22539850

11. Zhang $R$, Lahens NF, Ballance $H I$, Hughes $M E$, Hogenesch JB. A circadian gene expression atlas in mammals: implications for biology and medicine. Proc Natl Acad Sci U S A. 2014; 111:16219-24. https://doi.org/10.1073/pnas.1408886111 PMID: 25349387

12. Marcheva B, Ramsey KM, Buhr ED, Kobayashi Y, Su H, Ko $\mathrm{CH}$, Ivanova $\mathrm{G}$, Omura $\mathrm{C}$, Mo S, Vitaterna $\mathrm{MH}$, Lopez JP, Philipson LH, Bradfield CA, et al. Disruption of the clock components CLOCK and BMAL1 leads to hypoinsulinaemia and diabetes. Nature. 2010; 466:627-31.

https://doi.org/10.1038/nature09253 PMID:20562852

13. Curtis AM, Fitzgerald GA. Central and peripheral clocks in cardiovascular and metabolic function. Ann Med. 2006; 38:552-9.

https://doi.org/10.1080/07853890600995010 PMID:17438670

14. Musiek ES, Lim MM, Yang G, Bauer AQ, Qi L, Lee Y, Roh $\mathrm{JH}$, Ortiz-Gonzalez X, Dearborn JT, Culver JP, Herzog ED, Hogenesch JB, Wozniak DF, et al. Circadian clock proteins regulate neuronal redox homeostasis and neurodegeneration. J Clin Invest. 2013; 123:5389-400.
https://doi.org/10.1172/JCI70317 PMID:24270424

15. Li Y, Li G, Wang H, Du J, Yan J. Analysis of a gene regulatory cascade mediating circadian rhythm in zebrafish. PLoS Comput Biol. 2013; 9:e1002940. https://doi.org/10.1371/journal.pcbi.1002940 PMID: $\underline{23468616}$

16. Ramos BC, Moraes MN, Poletini MO, Lima LH, Castrucci AM. From blue light to clock genes in zebrafish ZEM-2S cells. PLoS One. 2014; 9:e106252. https://doi.org/10.1371/journal.pone.0106252 PMID:25184495

17. Liu J, Malkani G, Shi X, Meyer M, CunninghamRunddles S, Ma X, Sun ZS. The circadian clock Period 2 gene regulates gamma interferon production of NK cells in host response to lipopolysaccharide-induced endotoxic shock. Infect Immun. 2006; 74:4750-6.

https://doi.org/10.1128/IAI.00287-06

PMID:16861663

18. Zerbino DR, Achuthan $P$, Akanni W, Amode $M R$, Barrell D, Bhai J, Billis K, Cummins C, Gall A, Girón CG, Gil L, Gordon L, Haggerty L, et al. Ensembl 2018. Nucleic Acids Res. 2018; 46:D754-61. https://doi.org/10.1093/nar/gkx1098 PMID:29155950

19. Reichwald K, Petzold A, Koch P, Downie BR, Hartmann N, Pietsch S, Baumgart M, Chalopin D, Felder M, Bens M, Sahm A, Szafranski K, Taudien S, et al. Insights into Sex Chromosome Evolution and Aging from the Genome of a Short-Lived Fish. Cell. 2015; 163:1527-38.

https://doi.org/10.1016/i.cell.2015.10.071 PMID:26638077

20. Baumgart $M$, Barth $E$, Savino $A$, Groth $M$, Koch $P$, Petzold A, Arisi I, Platzer M, Marz M, Cellerino A. A miRNA catalogue and ncRNA annotation of the shortliving fish Nothobranchius furzeri. BMC Genomics. 2017; 18:693.

https://doi.org/10.1186/s12864-017-3951-8 PMID:28874118

21. Aramillo Irizar $P$, Schäuble $S$, Esser $D$, Groth $M$, Frahm C, Priebe S, Baumgart M, Hartmann N, Marthandan S, Menzel U, Müller J, Schmidt S, Ast V, et al. Transcriptomic alterations during ageing reflect the shift from cancer to degenerative diseases in the elderly. Nat Commun. 2018; 9:327. https://doi.org/10.1038/s41467-017-02395-2 PMID:29382830

22. World Health Organization. World health statistics 2016: monitoring health for the SDGs sustainable development goals. 2016. http://www.who.int/gho/ publications/world health statistics/2016/en/. 
23. Kail RV, Cavanaugh JC. Human development: A lifespan view (8th edition). Cengage Learning. 2018; 470-3.

24. Whitney CR. Jeanne Calment, World's Elder, Dies at 122. New York Times. 1997; Sect B: 8. https://www.nytimes.com/1997/08/05/world/jeanne -calment-world-s-elder-dies-at-122.html.

25. Pobojewski S. World's oldest mouse reaches milestone birthday. Medical School Communications. University of Michigan News Service. 2004. https://www.ur.umich.edu/0304/Apr19 04/26.shtml.

26. Rugh R. The mouse; its reproduction and development (1st edition). Minneapolis, Minn: Burgess Publishing Co. 1968.

27. Aires DJ, Rockwell G, Wang T, Frontera J, Wick J, Wang W, Tonkovic-Capin M, Lu J, E L, Zhu H, Swerdlow RH. Potentiation of dietary restrictioninduced lifespan extension by polyphenols. Biochim Biophys Acta. 2012; 1822:522-6.

https://doi.org/10.1016/i.bbadis.2012.01.005 PMID:22265987

28. Miller RA, Harper JM, Dysko RC, Durkee SJ, Austad $\mathrm{SN}$. Longer life spans and delayed maturation in wildderived mice. Exp Biol Med (Maywood). 2002; 227:500-8.

https://doi.org/10.1177/153537020222700715 PMID:12094015

29. Njiwa JRK, Müller P, Klein R. Life Cycle Stages and Length of Zebrafish (Danio rerio) Exposed to DDT. Journal of Health Science. 2004; 50:220-5.

https://doi.org/10.1248/ihs.50.220

30. Kishi S, Uchiyama J, Baughman AM, Goto T, Lin MC, Tsai SB. The zebrafish as a vertebrate model of functional aging and very gradual senescence. Exp Gerontol. 2003; 38:777-86.

https://doi.org/10.1016/s0531-5565(03)00108-6 PMID:12855287

31. Keller JM, Keller ET. The Use of Mature Zebrafish (Danio rerio) as a Model for Human Aging and Disease. Conn's Handbook of Models for Human Aging (Second Edition). 2018; 351-9. https://doi.org/10.1016/B978-0-12-811353-0.00026-9

32. Gerhard GS, Kauffman EJ, Wang X, Stewart R, Moore JL, Kasales CJ, Demidenko E, Cheng KC. Life spans and senescent phenotypes in two strains of Zebrafish (Danio rerio). Exp Gerontol. 2002; 37:1055-68. https://doi.org/10.1016/s0531-5565(02)00088-8 PMID:12213556

33. Valdesalici S, Cellerino A. Extremely short lifespan in the annual fish Nothobranchius furzeri. Proc Biol Sci. 2003 (Suppl 2); 270:S189-91. https://doi.org/10.1098/rsbl.2003.0048 PMID: 14667379

34. Terzibasi E, Valenzano DR, Benedetti M, Roncaglia $P$, Cattaneo A, Domenici L, Cellerino A. Large differences in aging phenotype between strains of the short-lived annual fish Nothobranchius furzeri. PLoS One. 2008; 3:e3866.

https://doi.org/10.1371/journal.pone.0003866 PMID:19052641

35. Vrtílek M, Žák J, Pšenička $M$, Reichard M. Extremely rapid maturation of a wild African annual fish. Curr Biol. 2018; 28:R822-4.

https://doi.org/10.1016/i.cub.2018.06.031 PMID:30086311

36. Schmieder R, Edwards R. Quality control and preprocessing of metagenomic datasets. Bioinformatics. 2011; 27:863-4. https://doi.org/10.1093/bioinformatics/btr026 PMID:21278185

37. Kim D, Pertea G, Trapnell C, Pimentel H, Kelley R, Salzberg SL. TopHat2: accurate alignment of transcriptomes in the presence of insertions, deletions and gene fusions. Genome Biol. 2013; 14:R36.

https://doi.org/10.1186/gb-2013-14-4-r36 PMID:23618408

38. Liao Y, Smyth GK, Shi W. featureCounts: an efficient general purpose program for assigning sequence reads to genomic features. Bioinformatics. 2014; 30:923-30.

https://doi.org/10.1093/bioinformatics/btt656 PMID:24227677

39. Wagner GP, Kin K, Lynch VJ. Measurement of mRNA abundance using RNA-seq data: RPKM measure is inconsistent among samples. Theory Biosci. 2012; 131:281-5.

https://doi.org/10.1007/s12064-012-0162-3 PMID:22872506

40. Love MI, Huber W, Anders S. Moderated estimation of fold change and dispersion for RNA-seq data with DESeq2. Genome Biol. 2014; 15:550. https://doi.org/10.1186/s13059-014-0550-8 PMID:25516281

41. Benjamini Y, Hochberg Y. Controlling the False Discovery Rate: a Practical and Powerful Approach to Multiple Testing. J R Stat Soc B. 1995; 57:289-300. https://www.jstor.org/stable/2346101.

42. van der Maaten L, Hinton G. Visualizing Data using tSNE. J Mach Learn Res. 2008; 9:2579-605.

43. Barth E, Srivastava A, Stojiljkovic M, Frahm C, Axer H, Witte OW, Marz M. Conserved aging-related 
signatures of senescence and inflammation in different tissues and species. Aging (Albany NY). 2019; 11:8556-72.

https://doi.org/10.18632/aging.102345

PMID:31606727

44. Srivastava A, Barth E, Ermolaeva MA, Guenther $M$, Frahm C, Marz M, Witte OW. Tissue-specific Gene Expression Changes Are Associated with Aging in Mice. Genomics Proteomics Bioinformatics. 2020; 18:430-42.

https://doi.org/10.1016/j.gpb.2020.12.001

PMID:33309863

45. Jonker MJ, Melis JP, Kuiper RV, van der Hoeven TV, Wackers PFK, Robinson J, van der Horst GT, Dollé ME, Vijg J, Breit TM, Hoeijmakers JH, van Steeg H. Life spanning murine gene expression profiles in relation to chronological and pathological aging in multiple organs. Aging Cell. 2013; 12:901-9.

https://doi.org/10.1111/acel.12118

PMID:23795901

46. Frøland Steindal IA, Whitmore D. Circadian Clocks in Fish-What Have We Learned so far? Biology (Basel). 2019; 8:17. https://doi.org/10.3390/biology8010017 PMID:30893815

47. Morf J, Rey G, Schneider K, Stratmann M, Fujita J, Naef F, Schibler U. Cold-inducible RNA-binding protein modulates circadian gene expression posttranscriptionally. Science. 2012; 338:379-83. https://doi.org/10.1126/science.1217726 PMID:22923437

48. Krylov VV, Izvekov El, Pavlova VV, Pankova NA, Osipova EA. Circadian rhythms in zebrafish (Danio rerio) behaviour and the sources of their variability. Biol Rev Camb Philos Soc. 2021; 96:785-97. https://doi.org/10.1111/brv.12678 PMID:33331134

49. Takumi T, Matsubara C, Shigeyoshi Y, Taguchi K, Yagita K, Maebayashi Y, Sakakida Y, Okumura K, Takashima N, Okamura H. A new mammalian period gene predominantly expressed in the suprachiasmatic nucleus. Genes Cells. 1998; 3:167-76.

https://doi.org/10.1046/j.1365-2443.1998.00178.x PMID: $\underline{9619629}$

50. Zheng B, Larkin DW, Albrecht U, Sun ZS, Sage M, Eichele G, Lee CC, Bradley A. The mPer2 gene encodes a functional component of the mammalian circadian clock. Nature. 1999; 400:169-73.

https://doi.org/10.1038/22118

PMID:10408444

51. Wood PA, Yang X, Hrushesky WJ. Clock genes and cancer. Integr Cancer Ther. 2009; 8:303-8. https://doi.org/10.1177/1534735409355292 PMID:20042409

52. Gery S, Gombart AF, Yi WS, Koeffler C, Hofmann WK, Koeffler HP. Transcription profiling of C/EBP targets identifies Per2 as a gene implicated in myeloid leukemia. Blood. 2005; 106:2827-36. https://doi.org/10.1182/blood-2005-01-0358 PMID:15985538

53. Hua $H$, Wang $Y$, Wan $C$, Liu $Y$, Zhu B, Yang C, Wang $X$, Wang Z, Cornelissen-Guillaume G, Halberg F. Circadian gene mPer2 overexpression induces cancer cell apoptosis. Cancer Sci. 2006; 97:589-96. https://doi.org/10.1111/j.1349-7006.2006.00225.x PMID: 16827798

54. Yang X, Wood PA, Ansell C, Hrushesky WJ. Circadian time-dependent tumor suppressor function of period genes. Integr Cancer Ther. 2009; 8:309-16. https://doi.org/10.1177/1534735409352083 PMID:19926612

55. Kalfalah F, Janke L, Schiavi A, Tigges J, Ix A, Ventura N, Boege $\mathrm{F}$, Reinke $\mathrm{H}$. Crosstalk of clock gene expression and autophagy in aging. Aging (Albany NY). 2016; 8:1876-95.

https://doi.org/10.18632/aging.101018 PMID:27574892

56. Martinez-Lopez N, Athonvarangkul D, Singh R. Autophagy and Aging. Longevity Genes. 2015; 73-87. https://doi.org/10.1007/978-1-4939-2404-2_3

57. Schiavi A, Torgovnick A, Kell A, Megalou E, Castelein N, Guccini I, Marzocchella L, Gelino S, Hansen M, Malisan F, Condò I, Bei R, Rea SL, et al. Autophagy induction extends lifespan and reduces lipid content in response to frataxin silencing in C. elegans. Exp Gerontol. 2013; 48:191-201.

https://doi.org/10.1016/i.exger.2012.12.002 PMID:23247094

58. Pyo JO, Yoo SM, Ahn HH, Nah J, Hong SH, Kam $\mathrm{TI}$, Jung $S$, Jung YK. Overexpression of Atg 5 in mice activates autophagy and extends lifespan. Nat Commun. 2013; 4:2300.

https://doi.org/10.1038/ncomms3300

PMID:23939249

59. Madeo F, Zimmermann A, Maiuri MC, Kroemer G. Essential role for autophagy in life span extension. J Clin Invest. 2015; 125:85-93.

https://doi.org/10.1172/JCl73946 PMID:25654554

60. Honma S, Kawamoto T, Takagi Y, Fujimoto K, Sato F, Noshiro M, Kato Y, Honma K. Dec1 and Dec2 are regulators of the mammalian molecular clock. 
Nature. 2002; 419:841-4.

https://doi.org/10.1038/nature01123

PMID:12397359

61. Kato $\mathrm{Y}$, Kawamoto $\mathrm{T}$, Fujimoto $\mathrm{K}$, Noshiro $\mathrm{M}$. DEC1/STRA13/SHARP2 and DEC2/SHARP1 coordinate physiological processes, including circadian rhythms in response to environmental stimuli. Curr Top Dev Biol. 2014; 110:339-72.

https://doi.org/10.1016/B978-0-12-405943-

$\underline{6.00010-5}$

PMID:25248482

62. Sato F, Bhawal UK, Yoshimura T, Muragaki Y. DEC1 and DEC2 Crosstalk between Circadian Rhythm and Tumor Progression. J Cancer. 2016; 7:153-9.

https://doi.org/10.7150/jca.13748

PMID:26819638

63. Guillaumond F, Lacoche S, Dulong S, Grechez-Cassiau A, Filipski E, Li XM, Lévi F, Berra E, Delaunay F, Teboul $M$. Altered Stra13 and Dec2 circadian gene expression in hypoxic cells. Biochem Biophys Res Commun. 2008; 369:1184-9.

https://doi.org/10.1016/i.bbrc.2008.03.009 PMID: 18342625

64. Liu Y, Sato F, Kawamoto T, Fujimoto K, Morohashi S, Akasaka H, Kondo J, Wu Y, Noshiro M, Kato Y, Kijima $\mathrm{H}$. Anti-apoptotic effect of the basic helix-loop-helix (bHLH) transcription factor DEC2 in human breast cancer cells. Genes Cells. 2010; 15:315-25. https://doi.org/10.1111/j.1365-2443.2010.01381.x PMID:20236182

65. Imaizumi $T$, Sato $F$, Tanaka H, Matsumiya $T$, Yoshida $\mathrm{H}$, Yashiro-Aizawa T, Tsuruga K, Hayakari R, Kijima H, Satoh K. Basic-helix-loop-helix transcription factor DEC2 constitutes negative feedback loop in IFN- $\beta$ mediated inflammatory responses in human mesangial cells. Immunol Lett. 2011; 136:37-43.

https://doi.org/10.1016/i.imlet.2010.11.009 PMID:21129405

66. Olkkonen J, Kouri VP, Hynninen J, Konttinen YT, Mandelin J. Differentially Expressed in Chondrocytes 2 (DEC2) Increases the Expression of IL-1 $\beta$ and Is Abundantly Present in Synovial Membrane in Rheumatoid Arthritis. PLoS One. 2015; 10:e0145279. https://doi.org/10.1371/journal.pone.0145279 PMID:26710124

67. Pouly D, Chenaux S, Martin V, Babis M, Koch R, Nagoshi E, Katanaev VL, Gachon F, Staub O. USP2-45 Is a Circadian Clock Output Effector Regulating Calcium Absorption at the Post-Translational Level. PLoS One. 2016; 11:e0145155.

https://doi.org/10.1371/journal.pone.0145155

PMID:26756164
68. Yan J, Wang H, Liu Y, Shao C. Analysis of gene regulatory networks in the mammalian circadian rhythm. PLoS Comput Biol. 2008; 4:e1000193.

https://doi.org/10.1371/journal.pcbi.1000193 PMID:18846204

69. Chen CY, Logan RW, Ma T, Lewis DA, Tseng GC, Sibille $E$, McClung CA. Effects of aging on circadian patterns of gene expression in the human prefrontal cortex. Proc Natl Acad Sci U S A. 2016; 113:206-11. https://doi.org/10.1073/pnas.1508249112 PMID:26699485

70. Lin E, Kuo PH, Liu YL, Yang AC, Kao CF, Tsai SJ. Effects of circadian clock genes and environmental factors on cognitive aging in old adults in a Taiwanese population. Oncotarget. 2017; 8:24088-98. https://doi.org/10.18632/oncotarget.15493 PMID:28412756

71. Liao $\mathrm{Y}$, Tong L, Tang $\mathrm{L}, \mathrm{Wu} \mathrm{S}$. The role of coldinducible RNA binding protein in cell stress response. Int J Cancer. 2017; 141:2164-73.

https://doi.org/10.1002/ijc.30833 PMID:28608439

72. Eghlidi DH, Luna SL, Brown DI, Garyfallou VT, Kohama SG, Urbanski HF. Gene expression profiling of the SCN in young and old rhesus macaques. J Mol Endocrinol. 2018; 61:57-67.

https://doi.org/10.1530/JME-18-0062 PMID:29743294

73. Pellegrino R, Kavakli IH, Goel N, Cardinale CJ, Dinges DF, Kuna ST, Maislin G, Van Dongen HP, Tufik S, Hogenesch JB, Hakonarson $\mathrm{H}$, Pack Al. A novel BHLHE41 variant is associated with short sleep and resistance to sleep deprivation in humans. Sleep. 2014; 37:1327-36.

https://doi.org/10.5665/sleep.3924 PMID: 25083013

74. Aunan JR, Cho WC, Søreide K. The Biology of Aging and Cancer: A Brief Overview of Shared and Divergent Molecular Hallmarks. Aging Dis. 2017; 8:628-42. https://doi.org/10.14336/AD.2017.0103 PMID:28966806

75. Kondratov RV, Kondratova AA, Gorbacheva VY, Vykhovanets OV, Antoch MP. Early aging and agerelated pathologies in mice deficient in BMAL1, the core componentof the circadian clock. Genes Dev. 2006; 20:1868-73.

https://doi.org/10.1101/gad.1432206 PMID: 16847346

76. Khapre RV, Kondratova AA, Susova O, Kondratov RV. Circadian clock protein BMAL1 regulates cellular senescence in vivo. Cell Cycle. 2011; 10:4162-9. https://doi.org/10.4161/cc.10.23.18381 PMID:22101268 
77. Keniry M, Dearth RK, Persans M, Parsons R. New Frontiers for the NFIL3 bZIP Transcription Factor in Cancer, Metabolism and Beyond. Discoveries (Craiova). 2014; 2:e15. https://doi.org/10.15190/d.2014.7 PMID:26539561

78. Yamaguchi S, Mitsui S, Yan L, Yagita K, Miyake S, Okamura $\mathrm{H}$. Role of DBP in the circadian oscillatory mechanism. Mol Cell Biol. 2000; 20:4773-81. https://doi.org/10.1128/MCB.20.13.4773-4781.2000 PMID:10848603

79. Ueda HR, Hayashi S, Chen W, Sano M, Machida M, Shigeyoshi $Y$, lino $M$, Hashimoto $S$. System-level identification of transcriptional circuits underlying mammalian circadian clocks. Nat Genet. 2005; 37:187-92.

https://doi.org/10.1038/ng1504

PMID: 15665827

80. Cadenas C, van de Sandt L, Edlund K, Lohr M, Hellwig $B$, Marchan R, Schmidt M, Rahnenführer J, Oster $H$, Hengstler JG. Loss of circadian clock gene expression is associated with tumor progression in breast cancer. Cell Cycle. 2014; 13:3282-91. https://doi.org/10.4161/15384101.2014.954454 PMID:25485508

81. Gachon F, Olela FF, Schaad O, Descombes P, Schibler $U$. The circadian PAR-domain basic leucine zipper transcription factors DBP, TEF, and HLF modulate basal and inducible xenobiotic detoxification. Cell Metab. 2006; 4:25-36.

https://doi.org/10.1016/i.cmet.2006.04.015 PMID:16814730

82. Gachon F, Fonjallaz P, Damiola F, Gos P, Kodama T, Zakany J, Duboule D, Petit B, Tafti M, Schibler U. The loss of circadian PAR bZip transcription factors results in epilepsy. Genes Dev. 2004; 18:1397-412.

https://doi.org/10.1101/gad.301404 PMID:15175240

83. Relógio $A$, Thomas $P$, Medina-Pérez $P$, Reischl $S$, Bervoets S, Gloc E, Riemer P, Mang-Fatehi S, Maier B, Schäfer R, Leser U, Herzel H, Kramer A, Sers C. Rasmediated deregulation of the circadian clock in cancer. PLoS Genet. 2014; 10:e1004338. https://doi.org/10.1371/journal.pgen.1004338 PMID:24875049

84. Lehmann R, Childs L, Thomas P, Abreu M, Fuhr L, Herzel $H$, Leser $U$, Relógio A. Assembly of a comprehensive regulatory network for the mammalian circadian clock: a bioinformatics approach. PLoS One. 2015; 10:e0126283. https://doi.org/10.1371/journal.pone.0126283 PMID:25945798
85. Subramaniam M, Hawse JR, Rajamannan NM, Ingle $\mathrm{JN}$, Spelsberg TC. Functional role of KLF10 in multiple disease processes. Biofactors. 2010; 36:8-18.

https://doi.org/10.1002/biof.67 PMID:20087894

86. Geuens T, Bouhy D, Timmerman V. The hnRNP family: insights into their role in health and disease. Hum Genet. 2016; 135:851-67. https://doi.org/10.1007/s00439-016-1683-5 PMID:27215579

87. Latorre E, Harries LW. Splicing regulatory factors, ageing and age-related disease. Ageing Res Rev. 2017; 36:165-70.

https://doi.org/10.1016/j.arr.2017.04.004 PMID:28456680

88. Lee BP, Pilling LC, Bandinelli S, Ferrucci L, Melzer D, Harries LW. The transcript expression levels of HNRNPM, HNRNPAO and AKAP17A splicing factors may be predictively associated with ageing phenotypes in human peripheral blood. Biogerontology. 2019; 20:649-63. https://doi.org/10.1007/s10522-019-09819-0 PMID: $\underline{31292793}$

89. Briata $\mathrm{P}, \mathrm{Chen} \mathrm{CY}$, Ramos A, Gherzi R. Functional and molecular insights into KSRP function in mRNA decay. Biochim Biophys Acta. 2013; 1829:689-94. https://doi.org/10.1016/j.bbagrm.2012.11.003 PMID:23178464

90. Gherzi R, Chen CY, Ramos A, Briata P. KSRP controls pleiotropic cellular functions. Semin Cell Dev Biol. 2014; 34:2-8.

https://doi.org/10.1016/i.semcdb.2014.05.004 PMID:24845017

91. Bollmann F, Art J, Henke J, Schrick K, Besche V, Bros M, Li H, Siuda D, Handler N, Bauer F, Erker T, Behnke F, Mönch B, et al. Resveratrol post-transcriptionally regulates pro-inflammatory gene expression via regulation of KSRP RNA binding activity. Nucleic Acids Res. 2014; 42:12555-69. https://doi.org/10.1093/nar/gku1033 PMID:25352548

92. Sato M, Stryker MP. Genomic imprinting of experience-dependent cortical plasticity by the ubiquitin ligase gene Ube3a. Proc Natl Acad Sci U S A. 2010; 107:5611-6.

https://doi.org/10.1073/pnas.1001281107 PMID:20212164

93. Sato M, Stryker MP. Distinctive features of adult ocular dominance plasticity. J Neurosci. 2008; 28:10278-86.

https://doi.org/10.1523/JNEUROSCI.2451-08.2008 PMID:18842887 
94. Williams K, Irwin DA, Jones DG, Murphy KM. Dramatic Loss of Ube3A Expression during Aging of the Mammalian Cortex. Front Aging Neurosci. 2010; 2:18.

https://doi.org/10.3389/fnagi.2010.00018 PMID:20559465

95. Niu $K$, Xiang L, Jin $Y$, Peng $Y$, Wu F, Tang W, Zhang $X$, Deng $H$, Xiang $H$, Li S, Wang J, Song $Q$, Feng $Q$. Identification of LARK as a novel and conserved Gquadruplex binding protein in invertebrates and vertebrates. Nucleic Acids Res. 2019; 47:7306-20. https://doi.org/10.1093/nar/gkz484 PMID:31165881

96. Kojima S, Matsumoto $K$, Hirose $M$, Shimada $M$, Nagano M, Shigeyoshi Y, Hoshino S, Ui-Tei K, Saigo K, Green CB, Sakaki Y, Tei H. LARK activates posttranscriptional expression of an essential mammalian clock protein, PERIOD1. Proc Natl Acad Sci U S A. 2007; 104:1859-64.

https://doi.org/10.1073/pnas.0607567104 PMID:17264215

97. Schittek B, Sinnberg T. Biological functions of casein kinase 1 isoforms and putative roles in tumorigenesis. Mol Cancer. 2014; 13:231. https://doi.org/10.1186/1476-4598-13-231 PMID:25306547

98. Bae JS, Park SH, Jamiyandorj U, Kim KM, Noh SJ, Kim JR, Park HJ, Kwon KS, Jung SH, Park HS, Park $\mathrm{BH}$, Lee $\mathrm{H}$, Moon WS, et al. CK2 $\alpha / C S N K 2 \mathrm{~A} 1$ Phosphorylates SIRT6 and Is Involved in the Progression of Breast Carcinoma and Predicts Shorter Survival of Diagnosed Patients. Am J Pathol. 2016; 186:3297-315.

https://doi.org/10.1016/i.ajpath.2016.08.007 PMID:27746184

99. Jawaid A, Woldemichael BT, Kremer EA, Laferriere F, Gaur N, Afroz T, Polymenidou M, Mansuy IM. Memory Decline and Its Reversal in Aging and Neurodegeneration Involve miR-183/96/182 Biogenesis. Mol Neurobiol. 2019; 56:3451-62. https://doi.org/10.1007/s12035-018-1314-3 PMID:30128653

100. Mason B, Laman H. The FBXL family of F-box proteins: variations on a theme. Open Biol. 2020; 10:200319.

https://doi.org/10.1098/rsob.200319

PMID: $\underline{3234069}$
101. Aging Atlas Consortium. Aging Atlas: a multi-omics database for aging biology. Nucleic Acids Res. 2021; 49:D825-30.

https://doi.org/10.1093/nar/gkaa894 PMID:33119753

102. Storch KF, Lipan O, Leykin I, Viswanathan N, Davis FC, Wong WH, Weitz CJ. Extensive and divergent circadian gene expression in liver and heart. Nature. 2002; 417:78-83.

https://doi.org/10.1038/nature744

PMID:11967526

103. Hojo H, Enya S, Arai M, Suzuki Y, Nojiri T, Kangawa K, Koyama S, Kawaoka S. Remote reprogramming of hepatic circadian transcriptome by breast cancer. Oncotarget. 2017; 8:34128-40.

https://doi.org/10.18632/oncotarget.16699 PMID:28388556

104. Vatine G, Vallone D, Appelbaum L, Mracek P, BenMoshe Z, Lahiri K, Gothilf Y, Foulkes NS. Light directs zebrafish period2 expression via conserved $D$ and $E$ boxes. PLoS Biol. 2009; 7:e1000223.

https://doi.org/10.1371/journal.pbio.1000223 PMID:19859524

105. Zhao H, Di Mauro G, Lungu-Mitea $S$, Negrini $P$, Guarino AM, Frigato $E$, Braunbeck $T$, Ma $H$, Lamparter T, Vallone D, Bertolucci C, Foulkes NS. Modulation of DNA Repair Systems in Blind Cavefish during Evolution in Constant Darkness. Curr Biol. 2018; 28:3229-43.e4.

https://doi.org/10.1016/j.cub.2018.08.039 PMID:30318355

106. Mracek P, Santoriello C, Idda ML, Pagano C, BenMoshe Z, Gothilf Y, Vallone D, Foulkes NS. Regulation of per and cry genes reveals a central role for the D-box enhancer in light-dependent gene expression. PLoS One. 2012; 7:e51278.

https://doi.org/10.1371/journal.pone.0051278 PMID:23236462

107. Pagano C, Siauciunaite R, Idda ML, Ruggiero G, Ceinos RM, Pagano M, Frigato E, Bertolucci C, Foulkes NS, Vallone D. Evolution shapes the responsiveness of the D-box enhancer element to light and reactive oxygen species in vertebrates. Sci Rep. 2018; 8:13180. https://doi.org/10.1038/s41598-018-31570-8 PMID:30181539 


\section{SUPPLEMENTARY MATERIALS}

\section{Supplementary Data}

All supplementary Data can be found at the Open Science Framework: https://osf.io/aydjt/. 